\title{
IMPACT OF BEHAVIOURAL ATTENTION ON THE HOUSEHOLDS' FOREIGN CURRENCY SAVINGS AS A RESPONSE TO THE EXTERNAL MACROECONOMIC SHOCKS
}

\section{Vilma Deltuvaitè, Svatopluk Kapounek, Petr Koráb*}

\begin{abstract}
This paper investigates the impact of behavioural attention on the households' foreign currency savings as a response to the external macroeconomic shocks. The information that the households acquire via different communication channels is expected to influence their decisions regarding their savings' allocation into different currencies. This study has applied the fundamental macroeconomic models by including individuals' attention to the specific risks and search interest in specific keywords on Google in order to assess the impact of acquired information and its communication channel on the households' foreign currency savings. We employed a twolevel mixed effects model including macroeconomic fundamentals and individuals' attention to the information determinants. We solved a problem of a long list of potential explanatory variables (keywords) by employing the Bayesian Model Averaging. This study assumes that households are more sensitive to the macroeconomic shocks (factors) if they search simultaneously for information on Google about these factors or specific related risks. The results emphasize the role behavioural attention during financial turmoil and economic downturn periods, especially in the environment of very low interest rates.
\end{abstract}

Keywords: foreign exchange, macroeconomic shocks, households, behavioural attention, Google Trends, Bayesian Model Averaging

JEL Classifications: F31, F62, D14, G11, C23, C11

\section{Introduction}

Traditional economic theory posits that individuals make investment decisions by maximizing a profit function and relying on macroeconomic fundamentals. However, the role of information and behavioural attention prevails in the environment of very low interest rates.

* Vilma Deltuvaite, Kaunas University of Technology, Kaunas, Lithuania (deltuvaite.v@ans.lt); Svatopluk Kapounek, Mendel University in Brno, Czech Republic, and Kaunas University of Technology, Kaunas, Lithuania (kapounek@mendelu.cz);

Petr Koráb, Mendel University in Brno, Czech Republic, and Kaunas University of Technology, Kaunas, Lithuania (petr.korab@mendelu.cz).

This research was funded by the Grant (No. MIP-016/2015) from the Research Council of Lithuania and the Grant No. 16-26353S "Sentiment and Its Impact on Stock Markets" from the Czech Science Foundation. We benefited from comments and suggestions made by the participants of the 19th International Conference Enterprise and Competitive Environment 2016 in Brno, March 2016, the Joint Annual Meeting of the Slovak Economic Association and the Austrian Economic Association in Bratislava, May 2016, the 5th Annual Lithuanian Conference on Economic Research in Vilnius, June 2016, the 6th International Conference of the Financial Engineering and Banking Society in Málaga, June 2016, the Annual Meeting of Academy of Behavioral Finance \& Economics in Las Vegas, September 2016, and the World Finance Conference in Sardinia, July, 2017. We are particularly grateful to Jarko Fidrmuc, Konstantins Benkovskis and Egle Karmazienè for their helpful comments and suggestions. 
Behavioural economists and policy makers are interested in how information that the households acquire via different communication channels influences their saving behaviour.

The importance of understanding the impact of behavioural attention on the households' foreign currency savings lies in a number of reasons. First, Schlueter et al. (2015) argue that a significant share of foreign currency savings creates a transmission channel of external monetary disturbances. External macroeconomic shocks increase the pressure on the monetary authorities to adjust these macroeconomic shocks using foreign reserves which in turns create the risk of national currency devaluation or speculative attacks regarding the national currency. Secondly, Allen and Saunders (2014) highlight that sudden decisions of households to reallocate savings in foreign currencies increase net foreign exchange position(s) of commercial banks resulting in an increase of foreign exchange risk. Thirdly, in Central and Eastern European countries (CEECs) the share of foreign currency deposits is significant. For example, in 2014 this share accounted for almost 40 per cent in Romania and almost 50 per cent in Bulgaria, while these countries are one of the most dependent economies on international remittances in the EU. In other CEECs (Hungary and Lithuania) the share of deposits in foreign currency is about 15-20 per cent, while in the Czech Republic, Poland, Estonia, and Latvia is less than 10 per cent. Fourthly, Olgu (2011) stresses that in selected CEECs (Latvia and Lithuania) the share of foreign currency deposits increased significantly in 2008-2009 in relation to the external macroeconomic shocks and domestic banking crisis suggesting that aggregate households' foreign currency savings are affected by different types of shocks. Finally, despite the importance of investigating and understanding the impact of behavioural attention on the households' foreign currency savings from a macroeconomic policy perspective, the behavioural attention and its impact on households' foreign currency savings has been investigated only in a few studies (Arifovic, 2001; Sharma et al., 2005; Bresser-Pereira et al., 2014; Kapounek et al., 2016b).

This paper essentially improves our knowledge about the impact of behavioural attention on aggregate households' foreign currency savings and contributes to the literature on the behavioural attention of individuals in a few ways. First, this empirical study is a first cross-country study investigating the impact of behavioural attention on aggregate households' foreign currency savings. Previous empirical studies on the households' decisions regarding their savings currency have focussed only on one or several countries (Arifovic, 2001; Sharma et al., 2005; Bresser-Pereira et al., 2014). Secondly, this study focuses not only on macroeconomic fundamentals, but also includes individuals' attention's determinants potentially influencing their preferences to savings currency. The behavioural attention aspects of the households' saving decisions have not been investigated so far to our knowledge. Thirdly, this empirical study applies a novel manner to investigate the impact of behavioural attention on aggregate households' foreign currency savings using Google Trends data. The empirical findings contribute to the literature on the individuals' behavioural attention explanation using Google Trends data (Da et al., 2011; Vlastakis and Markellos, 2012; Saxa, 2014; Irresberger et al., 2015; Yang et al., 2015; Kapounek et al., 2016a). Finally, this study provides new insights on the impact of behavioural 
attention on aggregate households' foreign currency savings and assumes that households are more sensitive to macroeconomic shocks (factors) if they search simultaneously for information on Google about these factors or specific related risks.

We employed a unique dataset constructed from domestic and foreign currency deposits' data, macroeconomic fundamentals, and Google's search keywords translated to different languages on 10 EU countries outside the euro area with their own currencies.

The empirical findings suggest that households' foreign currency savings are affected mostly by exchange rates movements and economic shocks, while the effect of inflation and interest rate shocks is very limited. We can assume that households' foreign currency savings could be influenced by individuals' more intense attention in specific risks and higher search interest on Google for specific keywords. The results emphasize the changes in the role of macroeconomic determinants on foreign currency savings during the times changing attention in these specific risks.

The organization of the paper is as follows. Section 2 provides theoretical background of the paper. Section 3 describes data and determinants used in macroeconomic models. Section 4 describes research strategy and methodology. Section 5 provides the empirical results, while Section 6 provides the results of robustness analysis. Section 7 discusses the main empirical findings and concludes.

\section{Theoretical Background}

While the paper investigates the impact of behavioural attention on aggregate households' foreign currency savings, the appropriate macroeconomic approach and model should be applied in order to determine the macroeconomic factors influencing households' foreign currency savings. Literature provides different macroeconomic approaches regarding the exchange rate determination (Boughton, 1988; Rogoff, 1996; Harvey, 1996; Nag and Mitra, 1998; Yuan, 2011).

The monetary approach originated from the traditional approach to the balance of payments explains the foreign exchange rate in terms of money supply and demand, level of real income, and interest rate both domestic and foreign using these variables for determination of the domestic and foreign price levels (Polak, 1957). According to the monetary approach, the exchange rate is determined by the ratio of the domestic and foreign price levels. A rise in foreign interest rate would lower the demand for cash of foreign currency and create an excess money supply of foreign currency, and consequently would cause a rise in foreign prices, followed by a depreciation of the foreign currency. However, the monetary approach does not incorporate the role of capital market in the exchange rate determination, despite the inclusion of interest rate, which role is limited to the effect on the price level.

In contrast to the monetary approach, the trade flows approach explains the foreign exchange rate in terms of international trade flows and current account. According to the trade flows approach, changes in monetary or fiscal policies or other shocks affect country's international trade flows through shifts in the terms of trade or in the relation between country's domestic consumption and output. The increased demand for imports will raise the demand for foreign exchange and consequently the exchange rate will tend to depreciate, 
and conversely. In general, a strengthening of country's current account balance will cause an appreciation of the exchange rate, and vice versa (Isard, 1978; Mussa, 1979; Kenen, 1985).

While the portfolio balance approach developed by Tobin (1969) explains exchange rates movements by international capital flows changes; the portfolio balance approach assumes that investors have three different investment opportunities: domestic currency, foreign bonds, and domestic bonds. Whereas domestic and foreign bonds are not perfect investment substitutes, therefore, domestic and foreign bonds' yields can differ. According to the portfolio balance approach, the changes of investor's portfolio structure are caused either by country's current account imbalances or government policy decisions. When a particular country experiences a current account deficit, it disposes of foreign bonds creating an excess supply of foreign bonds, consequently foreign currency will depreciate, and conversely. The depreciation of foreign currency will eliminate the country's current account surplus, and vice versa. According to the portfolio balance approach, the current account is the main driving force resulting exchange rate movements, while capital market readjustments are short-term and only in response to changes in the current account.

Investigation of the impact of behavioural attention on aggregate households' foreign currency savings is based on two leading macroeconomic models grounded on competing macroeconomic approaches to exchange rates: (1) Monetary Model presented by Mark (1995), and (2) Portfolio Balance Model introduced by Hooper and Morton (1982).

Behavioural economists (Tversky and Kahneman, 1981; Knoll, 2010; Lee et al., 2011 , etc.) argue that different form of the information leads to different investment decisions by individuals. Moreover, Stanovich and West (2000) emphasize that the interpretation of information by investment decision makers leads to investment decision differences. In summary, Knoll (2010) provides a number of empirical findings in the judgment and decision making as well as in behavioural economics literatures and demonstrates that various aspects of the decision context can significantly affect the households' savings.

We assume that internet search data explain behavioural attention of economic individuals in different risk factors. Moreover, the households can reallocate their savings according to the changes of their attention to the risks. In addition, the sensitivity of households on macroeconomic fundamentals changes according to the behavioural attention.

The information that the households acquire via different communication channels is expected to influence their savings' allocation into different currencies. For this reason, we will also extend the fundamental macroeconomic models by including individuals' attention to the specific risks and search interest in specific keywords on Google in order to assess the impact of acquired information and its communication channel on households' foreign currency savings.

The usefulness of internet search data in forecasting of economic indicators has been confirmed in many empirical studies (Bulut, 2015; Guzi and de Pedraza García, 2015; Kapounek et al., 2016a), while scientists have only recently begun to use the Internet search data in explanation of individuals' behaviour. Previous empirical studies provide 
substantial evidence about the usefulness of internet search data in explaining individuals' behaviour. Da et al. (2011) provided evidence that Google search data can capture investors' attention more efficiently comparing to other measures of investors' attention. Vlastakis and Markellos (2012) confirmed usefulness of Google Trends data for assessment of investors' information demand at the company and market level. Saxa (2014) examined the usefulness of Google Trends data for forecasting of individuals' behaviour and confirmed that internet search data improved forecasting results significantly. Irresberger et al. (2015) investigated investors' sentiments using Google Trends data and confirmed that some stock prices were significantly affected by investors' irrational sentiments irrespective of macroeconomic determinants. Yang et al. (2015) analysed the usefulness of search data for forecasting purposes of individuals' future behaviour and confirmed that search data significantly improved the forecasting performance. Kapounek et al. (2016a) provide evidence that individuals' behaviour was affected by their perception about specific risks and Google Trends data was useful in explaining individuals' saving decisions.

\section{Data}

The dataset in quarterly frequency covers $10 \mathrm{EU}$ countries outside the euro area with their own currencies: Bulgaria, the Czech Republic, Hungary, Poland, Romania, Sweden, the United Kingdom, Estonia, Latvia, and Lithuania for the period 2004 Q1-2014 Q4. Denmark was not included in the data sample due the data unavailability, while Croatia joined the EU only on $1^{\text {st }}$ July 2013. The ratio of households' deposits in foreign currency to total households' deposits ${ }^{1}$ is used as a dependent variable in all estimations, while macroeconomic determinants (regressors) are derived from two macroeconomic models explaining the exchange rates movements and described in Section 2. The definition of all macroeconomic determinants is presented in Appendix 1.

The dataset is completed with keywords from Google Trends database (the publicly available web facility provided by Google Inc.), which represents the frequency of searching information about specific keywords on Google. Google Trends data represents an index calculated as the frequency of particular searches on Google relative to the total search volumes. The dataset includes the Google Trends keywords in national languages reflecting different risks including geopolitical risk, political risk, financial risk, currency risk, credit risk, and general economic situation. A comprehensive list of Google Trends keywords including their translations into national languages is presented in Appendix 2.

\section{Methodology}

First, we provide baseline estimations of two leading macroeconomic models explaining the exchange rates: Monetary Model (MM) (1), and Portfolio Balance Model (PBM) (2). Foreign currency savings, $S A V E_{i t}$, represent share of aggregate households' deposits

In order to avoid the changes in foreign deposits caused by mechanical converting to national currency we used foreign deposits at the value of the exchange rate in the year 2009. 
in foreign currency to total households' deposits held at the monetary financial institutions in country $i$ and time $t$ :

$$
\begin{gathered}
S A V E_{i t}=\beta_{0}+\beta_{e} N E E R_{i t}+\beta_{m}\left(M_{i t}-M_{E A t}\right)+\beta_{g}\left(G D P_{i t}-G D P_{E A t}\right)+\mu_{i}+\theta_{t}+\varepsilon_{i t}, \\
S A V E_{i t}=\beta_{0}+\beta_{e} N E E R_{i t}+\beta_{r}\left(I R_{i t}-I R_{E A t}\right)+\beta_{p}\left(\pi_{i t}-\pi_{E A t}\right)+\beta_{m}\left(M_{i t}-M_{E A t}\right)+ \\
+\beta_{g}\left(G D P_{i t}-G D P_{E A t}\right)+\beta_{c} C A+\mu_{i}+\theta_{t}+\varepsilon_{i t},
\end{gathered}
$$

where variable NEER represents nominal effective exchange rate, $I R$ is a market interest rate, $\pi$ represents an inflation rate, variable $M$ is a monetary aggregate $\mathrm{M} 1, G D P$ represents economic activity, and variable $C A$ represents current account balance of a country $i$ at time $t$, including fixed effects $\beta$. All the differentials are calculated in relation to the euro area, denoted by $E A$. Finally, we include random effects $\mu$, time dummies $\theta$, and a possibly heteroscedastic residual $\varepsilon$. Thus, we consider a two-level mixed effects model with a random-intercept term at the country level. All presented macroeconomic models consider with non-lagged and lagged regressors (for one-quarter).

While the CEECs are the most dependent economies on international remittances in the EU and our data sample contains 8 CEECs, we have also modified the basic Portfolio Balance Model by including personal remittances instead of current account balance in Equation 2. Our data sample contains three Baltic countries that have recently adopted the euro (Estonia in 2011, Latvia in 2014, and Lithuania in 2015), therefore we deal with the problem of joining the Eurozone and fix the exchange rate on central parity.

Second, we provide augmented regressions and include individuals' attention to the information and search interest in specific keywords on Google related to the specific risks including geopolitical risk, political risk, financial risk, currency risk, credit risk, and general economic situation denoted by variable ATTENTION, which represents the frequency of searching information about specific keywords on Google by individuals. The selected keywords (Table A2) were identified as the most popular topics related to phrases "foreign saving", "foreign deposit", "domestic saving", and "domestic deposit". The keywords can be considered as specific information that individuals want to learn or it is a matter of their concerns:

$$
S A V E_{i t}=\beta_{0}+\sum_{s=1}^{S} \beta_{s} \operatorname{SHOCKS}_{s i t}+\sum_{l=1}^{L} \beta_{l} \text { ATTENTION }_{l i t}+\mu_{i}+\theta_{t}+\varepsilon_{i t},
$$

where the first set of variables, denoted by $S H O C K S$, represents selected macroeconomic determinants (shocks) specified in Equations (1)-(2). Due to the absence of the appropriate theoretical background we solve the problem of a long list of potential explanatory variables (keywords) and selection of the appropriate keywords of individuals' search interest by applying the Bayesian Model Averaging. Therefore, we reduce the uncertainty in the model by using Bayesian Model Averaging and estimate the probability of regressors to be included in the model. The Bayesian Model Averaging methodology is specified in Appendix 3. 
Third, we interacted macroeconomic determinants with dummies and differentiate between the low and the high individuals' attention to the keywords related to specific risks, where the higher attention is represented by searches over the average in the country $i$.

We apply maximum likelihood estimator and provide logs of likelihood statistics, fixed effects are tested by the Wald test and random effects for each country are tested by the likelihood-ratio test to compare the model with a one-level ordinary linear regression.

\section{Empirical Results}

First, we perform baseline estimates for three different macroeconomic models with non-lagged regressors (Models 1-3) and lagged regressors (Models 4-5) (Table 1). The empirical results of the baseline regressions' with non-lagged regressors indicate that macroeconomic determinants derived from the Monetary Model and Portfolio Balance Model are statistically significant in explaining households' foreign currency savings. While the basic and modified Portfolio Balance Models provide the most comprehensive explanation of the impact of macroeconomic determinants on households' foreign currency savings comparing to other macroeconomic models. The empirical findings suggest that GDP differential has a significant impact on the share of foreign currency deposits in all macroeconomic models. These empirical findings demonstrate that a more intense domestic economic growth motivates households to increase the share of deposits in national currency suggesting about increased confidence in national currency related with the improving economic situation in country. The appreciation of the national currency affects negatively returns on foreign currency deposits and causes reallocation in favour on deposits in national currency, and conversely. In addition, the higher inflation differential motivates households to save more in foreign currencies. On the contrary, we cannot confirm a robust significant impact of both the interest rate differential as well as money supply differential. In addition, we can state that households' foreign currency savings are affected mostly by economic shocks and exchange rates movements, while the effect of money supply and interest rate shocks is very limited.

The empirical findings of the baseline regressions' with lagged regressors confirm the negative effect of the increased money supply on share of foreign currency deposits. These results suggest that households prefer to save money for short-term consumption in national currency rather than in foreign currency due to the conversion costs or short-term exchange rate fluctuations.

The results of augmented regressions including individuals' attention to selected keywords related to the specific risks are presented in Table 2. The selected keywords were identified by posterior inclusion probability assessed using Bayesian Model Averaging (see Appendix 4). However, we can assume a significant impact only of nine keywords related to political risk ("political", "parliament", "premier"), financial risk ("money", "risk"), currency risk (currency code), unemployment risk ("employment"), credit risk ("debt"), and general economic situation ("growth"). 
Table 1 | Determinants of Households' Foreign Currency Savings: Baseline Regressions

\begin{tabular}{|c|c|c|c|c|c|c|}
\hline \multirow[b]{3}{*}{ Independent variables } & \multicolumn{6}{|c|}{$\begin{array}{l}\text { Dependent variable: foreign currency deposits } \\
\text { to total deposits (In) }\end{array}$} \\
\hline & \multicolumn{3}{|c|}{$\begin{array}{l}\text { Basic models with } \\
\text { non-lagged regressors }\end{array}$} & \multicolumn{3}{|c|}{$\begin{array}{l}\text { Basic models with } \\
\text { lagged regressors }\end{array}$} \\
\hline & MM & PBM & PBM (1) & MM & PBM & PBM (1) \\
\hline & (1) & (2) & (3) & (4) & (5) & (6) \\
\hline NEER & $\begin{array}{c}-0.288^{*} \\
(0.153)\end{array}$ & $\begin{array}{c}-0.343^{*} \\
(0.188)\end{array}$ & $\begin{array}{l}0.076 \\
(0.155)\end{array}$ & $\begin{array}{l}-0.260 \\
(0.167)\end{array}$ & $\begin{array}{c}-0.364^{* *} \\
(0.185)\end{array}$ & $\begin{array}{l}0.074 \\
(0.176)\end{array}$ \\
\hline$M 1_{\text {local }}-M 1_{E A}$ & $\begin{array}{c}0.077 \\
(0.239)\end{array}$ & $\begin{array}{c}0.277 \\
(0.382)\end{array}$ & $\begin{array}{c}0.215 \\
(0.233)\end{array}$ & $\begin{array}{c}-1.386^{* * *} \\
(0.262)\end{array}$ & $\begin{array}{c}0.057 \\
(0.377)\end{array}$ & $\begin{array}{c}-1.394^{* * *} \\
(0.266)\end{array}$ \\
\hline$G D P_{\text {local }}-G D P_{E A}$ & $\begin{array}{c}-2.776^{* * *} \\
(0.544)\end{array}$ & $\begin{array}{c}-2.329^{* * *} \\
(0.637)\end{array}$ & $\begin{array}{c}-1.991^{* * *} \\
(0.532)\end{array}$ & $\begin{array}{c}-2.337^{* * *} \\
(0.597)\end{array}$ & $\begin{array}{c}-2.496^{* * *} \\
(0.628)\end{array}$ & $\begin{array}{c}-1.950^{* * *} \\
(0.606)\end{array}$ \\
\hline$I R_{\text {local }}-I R_{E A}$ & - & $\begin{array}{l}-0.004 \\
(0.009)\end{array}$ & $\begin{array}{l}-0.010 \\
(0.007)\end{array}$ & - & $\begin{array}{l}-0.003 \\
(0.008)\end{array}$ & $\begin{array}{c}-0.019^{* *} \\
(0.008)\end{array}$ \\
\hline$\pi_{\text {local }}-\pi_{E A}$ & $\begin{array}{l}- \\
-\end{array}$ & $\begin{array}{l}0.008^{* * *} \\
(0.002)\end{array}$ & $\begin{array}{l}0.007^{* * *} \\
(0.001)\end{array}$ & - & $\begin{array}{l}0.010^{* * *} \\
(0.002)\end{array}$ & $\begin{array}{l}0.005^{* * *} \\
(0.001)\end{array}$ \\
\hline$C A$ & - & $\begin{array}{c}-0.073^{* * *} \\
(0.014)\end{array}$ & - & - & $\begin{array}{c}-0.064^{* * *} \\
(0.014)\end{array}$ & - \\
\hline REM & - & - & $\begin{array}{l}-0.020 \\
(0.031)\end{array}$ & - & - & $\begin{array}{l}-0.007 \\
(0.035)\end{array}$ \\
\hline Constant & $\begin{array}{l}-1.213 \\
(0.910)\end{array}$ & $\begin{array}{l}-0.870 \\
(1.056)\end{array}$ & $\begin{array}{c}-2.954^{* * *} \\
(0.891)\end{array}$ & $\begin{array}{l}-1.314 \\
(0.963)\end{array}$ & $\begin{array}{l}-0.824 \\
(1.041)\end{array}$ & $\begin{array}{c}-2.911^{* * *} \\
(0.974)\end{array}$ \\
\hline Random effects & Country & Country & Country & Country & Country & Country \\
\hline LRL & 88.21 & 71.40 & 115.2 & 57.06 & 74.86 & 71.25 \\
\hline Wald Test $\left(\chi^{2}\right)$ & 136.2 & 193.1 & 198.2 & 186.7 & 206.0 & 218.4 \\
\hline LR Test $\left(\bar{\chi}^{2}\right)$ & 1462 & 861.0 & 1281 & 1401 & 866.5 & 1199 \\
\hline Time dummies & YES & YES & YES & YES & YES & YES \\
\hline Number of observations & 350 & 234 & 346 & 350 & 234 & 346 \\
\hline Number of countries & 10 & 10 & 10 & 10 & 10 & 10 \\
\hline
\end{tabular}

Note: Standard errors in parentheses. ${ }^{* * *} p<0.01,{ }^{* *} p<0.05,{ }^{*} p<0.1$

Source: own calculation 
Table 2 | Determinants of Households' Foreign Currency Savings: Augmented Regressions

\begin{tabular}{|c|c|c|c|c|c|c|}
\hline \multirow{3}{*}{$\begin{array}{l}\text { Independent } \\
\text { variables }\end{array}$} & \multicolumn{6}{|c|}{ Dependent variable: foreign currency deposits to total deposits (In) } \\
\hline & \multicolumn{3}{|c|}{$\begin{array}{l}\text { Augmented models with non-lagged } \\
\text { regressors }\end{array}$} & \multicolumn{3}{|c|}{$\begin{array}{l}\text { Augmented models with lagged } \\
\text { regressors }\end{array}$} \\
\hline & MM & PBM & PBM (1) & MM & PBM & PBM (1) \\
\hline & (1) & (2) & (3) & (4) & (5) & (6) \\
\hline NEER & $\begin{array}{c}-0.520^{* * *} \\
(0.147)\end{array}$ & $\begin{array}{l}-0.098 \\
(0.168)\end{array}$ & $\begin{array}{c}-0.377^{* *} \\
(0.153)\end{array}$ & $\begin{array}{l}-0.584^{* * *} \\
(0.184)\end{array}$ & $\begin{array}{l}-0.138 \\
(0.166)\end{array}$ & $\begin{array}{c}-0.362^{*} \\
(0.197)\end{array}$ \\
\hline$M 1_{\text {local }}-M 1_{E A}$ & $\begin{array}{l}-0.073 \\
(0.191) \\
\end{array}$ & $\begin{array}{l}0.276 \\
(0.282) \\
\end{array}$ & $\begin{array}{c}0.016 \\
(0.188) \\
\end{array}$ & $\begin{array}{c}-1.675^{* * *} \\
(0.238) \\
\end{array}$ & $\begin{array}{l}-0.022 \\
(0.279) \\
\end{array}$ & $\begin{array}{c}-1.684^{* * *} \\
(0.242) \\
\end{array}$ \\
\hline$G D P_{\text {local }}-G D P_{E A}$ & $\begin{array}{c}-2.301^{* * *} \\
(0.432) \\
\end{array}$ & $\begin{array}{c}-2.113^{* * *} \\
(0.452) \\
\end{array}$ & $\begin{array}{c}-1.783^{* * *} \\
(0.430) \\
\end{array}$ & $\begin{array}{c}-2.115^{* * *} \\
(0.538) \\
\end{array}$ & $\begin{array}{c}-2.260^{* * *} \\
(0.447) \\
\end{array}$ & $\begin{array}{c}-1.856^{* * *} \\
(0.552) \\
\end{array}$ \\
\hline$I R_{\text {local }}-I R_{E A}$ & $\begin{array}{ll}- \\
-\end{array}$ & $\begin{array}{l}-0.004 \\
(0.006)\end{array}$ & $\begin{array}{c}0.001 \\
(0.006)\end{array}$ & $\begin{array}{ll}- \\
-\end{array}$ & $\begin{array}{l}-0.002 \\
(0.006)\end{array}$ & $\begin{array}{l}-0.010 \\
(0.008)\end{array}$ \\
\hline$\pi_{\text {local }}-\pi_{E A}$ & $\begin{array}{ll}- \\
-\end{array}$ & $\begin{array}{l}0.016^{* * *} \\
(0.002) \\
\end{array}$ & $\begin{array}{l}0.009^{* * * *} \\
(0.002) \\
\end{array}$ & $\begin{array}{ll}- \\
-\end{array}$ & $\begin{array}{l}0.019^{* * *} \\
(0.002)\end{array}$ & $\begin{array}{c}0.008^{* * *} \\
(0.002)\end{array}$ \\
\hline$C A$ & - & $\begin{array}{c}-0.044^{* * *} \\
(0.010) \\
\end{array}$ & - & - & $\begin{array}{c}-0.036^{* * *} \\
(0.010) \\
\end{array}$ & - \\
\hline REM & $\begin{array}{ll}- \\
-\end{array}$ & - & $\begin{array}{l}-0.013 \\
(0.025) \\
\end{array}$ & $\begin{array}{l}- \\
- \\
\end{array}$ & - & $\begin{array}{l}-0.011 \\
(0.033) \\
\end{array}$ \\
\hline Currency code & $\begin{array}{c}0.200^{* * *} \\
(0.032) \\
\end{array}$ & $\begin{array}{c}0.330^{* * *} \\
(0.041) \\
\end{array}$ & $\begin{array}{c}0.264^{* * *} \\
(0.033) \\
\end{array}$ & $\begin{array}{l}0.186^{* * *} \\
(0.040) \\
\end{array}$ & $\begin{array}{c}0.301^{* * *} \\
(0.040) \\
\end{array}$ & $\begin{array}{c}0.225^{* * *} \\
(0.042) \\
\end{array}$ \\
\hline “Debt” & $\begin{array}{c}0.021 \\
(0.025) \\
\end{array}$ & $\begin{array}{c}0.008 \\
(0.030) \\
\end{array}$ & $\begin{array}{l}0.053^{* *} \\
(0.025) \\
\end{array}$ & $\begin{array}{l}0.059^{*} \\
(0.032) \\
\end{array}$ & $\begin{array}{c}0.028 \\
(0.030) \\
\end{array}$ & $\begin{array}{c}0.094^{* * *} \\
(0.032) \\
\end{array}$ \\
\hline “Money" & $\begin{array}{l}0.137^{* *} \\
(0.061)\end{array}$ & $\begin{array}{l}0.119^{*} \\
(0.067) \\
\end{array}$ & $\begin{array}{c}0.154^{* * *} \\
(0.059)\end{array}$ & $\begin{array}{l}-0.018 \\
(0.076)\end{array}$ & $\begin{array}{l}0.145^{* *} \\
(0.066)\end{array}$ & $\begin{array}{l}-0.010 \\
(0.076) \\
\end{array}$ \\
\hline “Growth" & $\begin{array}{c}-0.215^{* * *} \\
(0.028) \\
\end{array}$ & $\begin{array}{c}-0.153^{* * *} \\
(0.029) \\
\end{array}$ & $\begin{array}{c}-0.182^{* * *} \\
(0.027) \\
\end{array}$ & $\begin{array}{c}-0.163^{* * *} \\
(0.035) \\
\end{array}$ & $\begin{array}{c}-0.143^{* * *} \\
(0.029) \\
\end{array}$ & $\begin{array}{c}-0.135^{* * *} \\
(0.035) \\
\end{array}$ \\
\hline "Employment" & $\begin{array}{c}-0.116^{* * *} \\
(0.025) \\
\end{array}$ & $\begin{array}{c}-0.226^{* * *} \\
(0.037) \\
\end{array}$ & $\begin{array}{c}-0.088^{* * *} \\
(0.024) \\
\end{array}$ & $\begin{array}{c}-0.067^{* *} \\
(0.031) \\
\end{array}$ & $\begin{array}{c}-0.228^{* * *} \\
(0.037) \\
\end{array}$ & $\begin{array}{l}-0.038 \\
(0.031) \\
\end{array}$ \\
\hline “Political” & $\begin{array}{l}-0.037 \\
(0.044) \\
\end{array}$ & $\begin{array}{l}0.150^{* * *} \\
(0.055) \\
\end{array}$ & $\begin{array}{c}0.022 \\
(0.044) \\
\end{array}$ & $\begin{array}{l}-0.011 \\
(0.055) \\
\end{array}$ & $\begin{array}{l}0.181^{* * *} \\
(0.054) \\
\end{array}$ & $\begin{array}{c}0.036 \\
(0.057) \\
\end{array}$ \\
\hline "Parliament" & $\begin{array}{c}0.046 \\
(0.039) \\
\end{array}$ & $\begin{array}{c}0.005 \\
(0.043) \\
\end{array}$ & $\begin{array}{c}0.052 \\
(0.038) \\
\end{array}$ & $\begin{array}{l}-0.008 \\
(0.049) \\
\end{array}$ & $\begin{array}{c}0.013 \\
(0.043) \\
\end{array}$ & $\begin{array}{c}0.000 \\
(0.049) \\
\end{array}$ \\
\hline “Premier” & $\begin{array}{c}-0.058^{* *} \\
(0.026) \\
\end{array}$ & $\begin{array}{c}-0.067^{* *} \\
(0.030) \\
\end{array}$ & $\begin{array}{c}-0.083^{* * *} \\
(0.026) \\
\end{array}$ & $\begin{array}{l}-0.063^{*} \\
(0.033) \\
\end{array}$ & $\begin{array}{c}-0.060^{* *} \\
(0.030) \\
\end{array}$ & $\begin{array}{c}-0.079 * * \\
(0.033) \\
\end{array}$ \\
\hline “Risk" & $\begin{array}{c}-0.147^{* * *} \\
(0.048)\end{array}$ & $\begin{array}{c}0.005 \\
(0.057)\end{array}$ & $\begin{array}{l}-0.048 \\
(0.050)\end{array}$ & $\begin{array}{l}-0.110^{*} \\
(0.060)\end{array}$ & $\begin{array}{l}-0.042 \\
(0.057)\end{array}$ & $\begin{array}{l}-0.027 \\
(0.065)\end{array}$ \\
\hline Constant & $\begin{array}{c}0.022 \\
(0.902)\end{array}$ & $\begin{array}{c}-3.167^{* * *} \\
(1.038)\end{array}$ & $\begin{array}{l}-1.759^{*} \\
(0.952)\end{array}$ & $\begin{array}{c}0.594 \\
(1.062)\end{array}$ & $\begin{array}{c}-3.107^{* * *} \\
(1.024)\end{array}$ & $\begin{array}{l}-1.317 \\
(1.162)\end{array}$ \\
\hline Random effects & Country & Country & Country & Country & Country & Country \\
\hline LRL & 177.2 & 154.3 & 192.3 & 103.6 & 156.8 & 109.4 \\
\hline Wald Test $\left(\chi^{2}\right)$ & 485.9 & 691.7 & 534.5 & 367.2 & 711.5 & 375.2 \\
\hline LR Test $\left(\bar{\chi}^{2}\right)$ & 1029 & 666.1 & 981.7 & 884.6 & 666.9 & 823.2 \\
\hline Time dummies & YES & YES & YES & YES & YES & YES \\
\hline $\begin{array}{l}\text { Number } \\
\text { of observations }\end{array}$ & 343 & 227 & 339 & 343 & 227 & 339 \\
\hline Number of countries & 10 & 10 & 10 & 10 & 10 & 10 \\
\hline
\end{tabular}

Note: Standard errors in parentheses. ${ }^{* * *} p<0.01,{ }^{* *} p<0.05,{ }^{*} p<0.1$

Source: own calculation 
The empirical estimations of augmented regressions' with non-lagged regressors provide evidence that macroeconomic determinants derived from both the basic and modified Portfolio Balance Models are significant in explaining households' foreign currency savings. In addition, the empirical findings suggest that inflation differential has a statistically significant impact on the share of foreign currency deposits in all macroeconomic models. These results suggest that a higher inflation differential motivate households to prefer saving in foreign currencies due to the decreasing real return on domestic currency deposits.

The results of augmented regressions' with non-lagged regressors confirm a robust significant impact of three keywords such as currency code, "growth", and "premier" in all macroeconomic models. The provided empirical estimations allow us to assume that exchange rates movements and more intense domestic economic growth have a significant impact on the share of foreign currency deposits in all macroeconomic models due to the individuals' more intense attention and higher search interest in currency code and keyword "growth" in Google respectively. In addition, individuals' higher search interest in currency code has a significant positive effect on the share of foreign currency deposits, while an opposite effect of search interest in keywords "growth" and "premier" was identified.

Thus, we can summarize that aggregate households' foreign currency savings could be influenced by individuals' more intense attention in specific risks and higher search interest in Google for specific keywords. We can assume that households' reaction to the external macroeconomic shocks (exchange rates and economic shocks) could be affected by their search activities in Google. These empirical findings suggest that the communication strategy (including the communication channels) of the public authorities during financial turmoil and economic downturn periods could affect aggregate households' foreign currency savings. The results of augmented regressions' with lagged regressors confirmed the empirical findings of the augmented regressions' with non-lagged regressors.

Table 3 demonstrates different impact of macroeconomic shocks on the households' foreign currency savings if individuals' attention (frequency of searches) to specific risks and specific keywords on Google is above or below the average. The previous empirical results did not provide a significant and robust effect of interest rate differential on the share of foreign currency deposits. The empirical estimations of augmented Portfolio Balance Model's confirm that interest rate differential is a significant macroeconomic determinant resulting households' deposits reallocation if frequency of searches in keyword "money" and currency code exceeds the average. These empirical findings suggest that events related to the national currency changes and disclosed by the media via internet channel increase the attention of households and motivate households to reallocate their deposits in relation to interest rate differential changes. In addition, these findings suggest that a higher interest rate differential motivate households to prefer saving in national currency rather than in foreign currencies due to the earning motives.

The regression results of augmented Portfolio Balance Model's provide substantial evidence that aggregate households' foreign currency savings are affected by exchange rate movements, more intense domestic economic growth, higher inflation differential, and changes in domestic current account balance despite the level of their attention. However, we assume that increased 
individuals' attention to the specific risks and more intense search interest on Google plays a crucial role in the foreign currency deposits reallocation and emphasizes the role of policy makers' communication strategy, especially in the environment of very low interest rates.

\section{Table 3 | Determinants of Households' Foreign Currency Savings: Augmented Portfolio Balance} Model

\begin{tabular}{|c|c|c|c|c|c|c|c|c|c|}
\hline & \multicolumn{9}{|c|}{ Dependent variable: foreign currency deposits to total deposits (In) } \\
\hline & \multicolumn{9}{|c|}{ Google keywords } \\
\hline & $\begin{array}{l}\text { Currency } \\
\text { code }\end{array}$ & “Debt” & "Money" & "Growth" & $\begin{array}{l}\text { "Employ- } \\
\text { ment" }\end{array}$ & "Political" & $\begin{array}{l}\text { "Parlia- } \\
\text { ment" }\end{array}$ & "Premier" & “Risk" \\
\hline $\begin{array}{l}\text { Independent } \\
\text { variables }\end{array}$ & (1) & (2) & (3) & (4) & (5) & (6) & (7) & (8) & (9) \\
\hline $\operatorname{NEER}(>\mathrm{A})$ & $\begin{array}{c}-0.782^{* * *} \\
(0.181)\end{array}$ & $\begin{array}{c}-0.449^{* *} \\
(0.190)\end{array}$ & $\begin{array}{c}-0.444^{* *} \\
(0.198)\end{array}$ & $\begin{array}{l}-0.078 \\
(0.167)\end{array}$ & $\begin{array}{l}-0.289 \\
(0.191)\end{array}$ & $\begin{array}{c}-0.419 * * \\
(0.177)\end{array}$ & $\begin{array}{c}-0.347^{*} \\
(0.181)\end{array}$ & $\begin{array}{l}-0.249 \\
(0.217)\end{array}$ & $\begin{array}{c}-0.410^{* *} \\
(0.184)\end{array}$ \\
\hline $\operatorname{NEER}(<A)$ & $\begin{array}{c}-0.637^{* * *} \\
(0.171)\end{array}$ & $\begin{array}{c}-0.402^{* *} \\
(0.185)\end{array}$ & $\begin{array}{c}-0.408^{* *} \\
(0.190)\end{array}$ & $\begin{array}{l}-0.044 \\
(0.169) \\
\end{array}$ & $\begin{array}{l}-0.264 \\
(0.196)\end{array}$ & \begin{tabular}{|c|}
$-0.477^{* * *}$ \\
$(0.182)$ \\
\end{tabular} & $\begin{array}{c}-0.382^{* *} \\
(0.185)\end{array}$ & $\begin{array}{l}-0.341 \\
(0.211) \\
\end{array}$ & $\begin{array}{c}-0.420^{* *} \\
(0.188)\end{array}$ \\
\hline$M 1_{\text {loc }}-M 1_{E A}(>A)$ & $\begin{array}{l}-0.279 \\
(0.531) \\
\end{array}$ & $\begin{array}{l}-0.574 \\
(0.505) \\
\end{array}$ & $\begin{array}{c}0.156 \\
(0.477) \\
\end{array}$ & $\begin{array}{l}-0.316 \\
(0.421) \\
\end{array}$ & $\begin{array}{c}0.245 \\
(0.467) \\
\end{array}$ & $\begin{array}{c}0.294 \\
(0.406) \\
\end{array}$ & $\begin{array}{c}0.379 \\
(0.423) \\
\end{array}$ & $\begin{array}{l}-0.180 \\
(0.503) \\
\end{array}$ & $\begin{array}{c}0.237 \\
(0.444) \\
\end{array}$ \\
\hline$M 1_{\text {loc }}-M 1_{E A}(<A)$ & $\begin{array}{c}0.649 \\
(0.418)\end{array}$ & $\begin{array}{l}1.123^{* *} \\
(0.464)\end{array}$ & $\begin{array}{l}0.999^{* *} \\
(0.463)\end{array}$ & $\begin{array}{l}-0.210 \\
(0.452)\end{array}$ & $\begin{array}{c}0.518 \\
(0.543)\end{array}$ & $\begin{array}{c}0.492 \\
(0.590)\end{array}$ & $\begin{array}{c}0.143 \\
(0.543)\end{array}$ & $\begin{array}{c}0.026 \\
(0.447)\end{array}$ & $\begin{array}{c}0.451 \\
(0.499)\end{array}$ \\
\hline$G D P_{l o c}-G D P_{E A}(>A)$ & $\begin{array}{c}-3.810^{* * *} \\
(0.813)\end{array}$ & $\begin{array}{c}-1.935^{*} \\
(1.102) \\
\end{array}$ & $\begin{array}{l}-1.292 \\
(0.882) \\
\end{array}$ & $\begin{array}{c}-2.297^{* *} \\
(0.923)\end{array}$ & $\begin{array}{c}-1.976^{* *} \\
(0.946)\end{array}$ & $\begin{array}{c}-1.463^{* *} \\
(0.741)\end{array}$ & $\begin{array}{c}-1.693^{* *} \\
(0.804)\end{array}$ & \begin{tabular}{|c|}
$-3.540^{* * *}$ \\
$(1.065)$ \\
\end{tabular} & $\begin{array}{c}-1.782^{*} \\
(0.969) \\
\end{array}$ \\
\hline$G D P_{l o c}-G D P_{E A}(<A)$ & $\begin{array}{l}-0.354 \\
(0.857) \\
\end{array}$ & $\begin{array}{c}-2.482^{* * *} \\
(0.748)\end{array}$ & $\begin{array}{c}-2.748^{* * *} \\
(0.800) \\
\end{array}$ & $\begin{array}{c}-1.462^{* *} \\
(0.720) \\
\end{array}$ & $\begin{array}{c}-2.674^{* * *} \\
(0.824) \\
\end{array}$ & \begin{tabular}{|c|}
$-3.941^{* * *}$ \\
$(0.904)$ \\
\end{tabular} & $\begin{array}{c}-3.303^{* * *} \\
(0.871) \\
\end{array}$ & $\begin{array}{c}-1.291^{*} \\
(0.705) \\
\end{array}$ & $\begin{array}{c}-2.549 * * * \\
(0.776)\end{array}$ \\
\hline$I R_{\text {loc }}-I R_{E A}(>A)$ & $\begin{array}{c}-0.039 * * * \\
(0.009)\end{array}$ & $\begin{array}{l}-0.011 \\
(0.010)\end{array}$ & $\begin{array}{c}-0.026^{* *} \\
(0.012)\end{array}$ & $\begin{array}{l}-0.001 \\
(0.009)\end{array}$ & $\begin{array}{l}-0.004 \\
(0.011)\end{array}$ & $\begin{array}{c}0.011 \\
(0.009)\end{array}$ & $\begin{array}{c}0.006 \\
(0.009)\end{array}$ & $\begin{array}{c}0.001 \\
(0.009)\end{array}$ & $\begin{array}{l}-0.001 \\
(0.010) \\
\end{array}$ \\
\hline$I R_{\text {loc }}-I R_{E A}(<A)$ & $\begin{array}{c}0.014 \\
(0.009)\end{array}$ & $\begin{array}{c}0.010 \\
(0.010)\end{array}$ & $\begin{array}{c}0.014 \\
(0.010)\end{array}$ & $\begin{array}{l}0.016^{*} \\
(0.009)\end{array}$ & $\begin{array}{c}0.000 \\
(0.010)\end{array}$ & \begin{tabular}{|c|}
$-0.049 * * *$ \\
$(0.012)$ \\
\end{tabular} & $\begin{array}{c}-0.042^{* * *} \\
(0.012)\end{array}$ & $\begin{array}{l}-0.008 \\
(0.011) \\
\end{array}$ & $\begin{array}{l}-0.013 \\
(0.009)\end{array}$ \\
\hline$\pi_{10 c}-\pi_{E A}(>A)$ & $\begin{array}{l}0.008^{* * *} \\
(0.002)\end{array}$ & $\begin{array}{l}0.009^{* * * *} \\
(0.002)\end{array}$ & $\begin{array}{l}0.011^{* * *} \\
(0.003)\end{array}$ & $\begin{array}{l}0.006^{* * *} \\
(0.002)\end{array}$ & $\begin{array}{l}0.011 * * * \\
(0.003)\end{array}$ & $\begin{array}{l}0.006^{* *} \\
(0.003)\end{array}$ & $\begin{array}{l}0.007^{* *} \\
(0.003)\end{array}$ & $\begin{array}{c}0.007^{* * *} \\
(0.002)\end{array}$ & $\begin{array}{c}0.009^{* * *} \\
(0.003)\end{array}$ \\
\hline$\pi_{\text {loc }}-\pi_{E A}(<A)$ & $\begin{array}{c}0.001 \\
(0.003) \\
\end{array}$ & $\begin{array}{c}0.007^{* * *} \\
(0.002)\end{array}$ & $\begin{array}{c}0.008^{* * *} \\
(0.003)\end{array}$ & $\begin{array}{c}0.000 \\
(0.002) \\
\end{array}$ & $\begin{array}{c}0.008^{* * *} \\
(0.002)\end{array}$ & $\begin{array}{c}0.010^{* * *} \\
(0.002)\end{array}$ & $\begin{array}{c}0.008^{* * *} \\
(0.003)\end{array}$ & $\begin{array}{c}0.010^{* * *} \\
(0.002)\end{array}$ & $\begin{array}{c}0.006^{* * *} \\
(0.002)\end{array}$ \\
\hline$C A(>A)$ & $\begin{array}{c}-0.038^{* * *} \\
(0.014)\end{array}$ & $\begin{array}{c}-0.043^{* *} \\
(0.018)\end{array}$ & $\begin{array}{c}-0.056^{* * *} \\
(0.019)\end{array}$ & $\begin{array}{c}-0.043^{* * *} \\
(0.016)\end{array}$ & $\begin{array}{c}-0.067^{* * *} \\
(0.018)\end{array}$ & \begin{tabular}{|c|}
$-0.112^{* * *}$ \\
$(0.019)$ \\
\end{tabular} & $\begin{array}{c}-0.113^{* * *} \\
(0.020)\end{array}$ & \begin{tabular}{|c|}
$-0.121^{* * *}$ \\
$(0.020)$ \\
\end{tabular} & $\begin{array}{c}-0.100^{* * *} \\
(0.024)\end{array}$ \\
\hline$C A(<A)$ & $\begin{array}{c}-0.177^{* * *} \\
(0.023)\end{array}$ & $\begin{array}{c}-0.088^{* * *} \\
(0.016)\end{array}$ & $\begin{array}{c}-0.082^{* * *} \\
(0.016)\end{array}$ & $\begin{array}{c}-0.030 * \\
(0.017)\end{array}$ & $\begin{array}{c}-0.079 * * * \\
(0.018)\end{array}$ & \begin{tabular}{|c|}
$-0.051^{* * *}$ \\
$(0.015)$ \\
\end{tabular} & $\begin{array}{c}-0.059^{* * *} \\
(0.015)\end{array}$ & \begin{tabular}{|c|}
$-0.042^{* * *}$ \\
$(0.015)$ \\
\end{tabular} & $\begin{array}{c}-0.062^{* * *} \\
(0.015)\end{array}$ \\
\hline Constant & $\begin{array}{r}1.089 \\
(1.033)\end{array}$ & $\begin{array}{l}-0.507 \\
(1.051)\end{array}$ & $\begin{array}{l}-0.510 \\
(1.073)\end{array}$ & $\begin{array}{c}-2.163^{* *} \\
(0.973)\end{array}$ & $\begin{array}{l}-1.235 \\
(1.079)\end{array}$ & $\begin{array}{l}-0.300 \\
(1.031)\end{array}$ & $\begin{array}{l}-0.717 \\
(1.040)\end{array}$ & $\begin{array}{l}-1.083 \\
(1.159)\end{array}$ & $\begin{array}{l}-0.489 \\
(1.055)\end{array}$ \\
\hline Random effects & Country & Country & Country & Country & Country & Country & Country & Country & Country \\
\hline LRL & 99.68 & 79.51 & 84.11 & 106.4 & 73.25 & 87.78 & 82.60 & 85.52 & 80.15 \\
\hline Wald Test $\left(\chi^{2}\right)$ & 313.7 & 224.4 & 242.9 & 346.3 & 199.9 & 259.1 & 237.3 & 249.2 & 227.3 \\
\hline LR Test $\left(\bar{\chi}^{2}\right)$ & 868.7 & 868.9 & 879.2 & 913.0 & 846.5 & 868.5 & 860.1 & 835.1 & 839.9 \\
\hline Time dummies & YES & YES & YES & YES & YES & YES & YES & YES & YES \\
\hline $\begin{array}{l}\text { Number } \\
\text { of observations }\end{array}$ & 234 & 234 & 234 & 234 & 234 & 234 & 234 & 234 & 234 \\
\hline $\begin{array}{l}\text { Number } \\
\text { of countries }\end{array}$ & 10 & 10 & 10 & 10 & 10 & 10 & 10 & 10 & 10 \\
\hline
\end{tabular}

Note: Standard errors in parentheses. ${ }^{* * *} p<0.01,{ }^{* *} p<0.05,{ }^{*} p<0.1$. $>$ A denotes the cases when frequency of searching in events is above the average, while $<$ A denotes the cases when frequency of searching in events is below the average.

Source: own calculation 


\section{Robustness Analysis}

Tables 4 to 5 show the results of our robustness checks in relation to the positive (Tables 4) and negative (Table 5) changes in share of foreign currency deposits. The results of robustness analysis presented in Tables 4 to 5 are very similar suggesting about the robustness of augmented Portfolio Balance Model's results in relation to the positive and negative changes in share of foreign currency deposits. However, we can state that increased individuals' attention to the specific risks and search interest for specific keywords on Google results negative changes in share of foreign currency deposits. These empirical results suggest that households are more confident in national currency in the context of external macroeconomic shocks.

In addition, we can state that interest rate differential is a significant macroeconomic determinant affecting aggregate households' foreign currency savings when individuals increase search attention in keywords such as currency code, money. These results suggest that households take into account the differences in interest rates only if they search simultaneously for information on Google on exchanges rate changes. We can also state that the reaction of households to the information related to the political situation in country is not affecting their saving decisions. 
Table 4 | Robustness of Augmented Portfolio Balance Model: Positive Changes of Foreign Currency Deposits' Ratio

\begin{tabular}{|c|c|c|c|c|c|c|c|c|c|}
\hline & \multicolumn{9}{|c|}{ Dependent variable: $\Delta$ of foreign currency deposits to total deposits $(\ln )(\Delta>0)$} \\
\hline & \multicolumn{9}{|c|}{ Google keywords } \\
\hline & \begin{tabular}{c|} 
Currency \\
code
\end{tabular} & “Debt” & "Money" & "Growth" & $\begin{array}{c}\text { "Employ- } \\
\text { ment" }\end{array}$ & "Political" & $\begin{array}{l}\text { "Parlia- } \\
\text { ment" }\end{array}$ & "Premier" & “Risk" \\
\hline $\begin{array}{l}\text { Independent } \\
\text { variables }\end{array}$ & (1) & (2) & (3) & (4) & (5) & (6) & (7) & (8) & (9) \\
\hline $\operatorname{NEER}(>\mathrm{A})$ & $\begin{array}{l}-0.404 \\
(0.282) \\
\end{array}$ & $\begin{array}{l}-0.261 \\
(0.279)\end{array}$ & $\begin{array}{l}-0.246 \\
(0.402)\end{array}$ & $\begin{array}{c}0.230 \\
(0.250) \\
\end{array}$ & $\begin{array}{c}0.101 \\
(0.290)\end{array}$ & $\begin{array}{l}-0.115 \\
(0.303)\end{array}$ & $\begin{array}{c}0.123 \\
(0.302)\end{array}$ & $\begin{array}{l}-0.254 \\
(0.349) \\
\end{array}$ & $\begin{array}{l}0.096 \\
(0.301) \\
\end{array}$ \\
\hline $\operatorname{NEER}(<\mathrm{A})$ & $\begin{array}{l}-0.216 \\
(0.264) \\
\end{array}$ & $\begin{array}{l}-0.205 \\
(0.272) \\
\end{array}$ & $\begin{array}{l}-0.161 \\
(0.374) \\
\end{array}$ & $\begin{array}{l}0.256 \\
(0.246) \\
\end{array}$ & $\begin{array}{c}0.152 \\
(0.297) \\
\end{array}$ & $\begin{array}{l}-0.194 \\
(0.319) \\
\end{array}$ & $\begin{array}{l}0.047 \\
(0.312) \\
\end{array}$ & $\begin{array}{l}-0.311 \\
(0.331) \\
\end{array}$ & $\begin{array}{l}0.066 \\
(0.308) \\
\end{array}$ \\
\hline$M 1_{\text {loc }}-M 1_{E A}(>A)$ & $\begin{array}{l}-0.312 \\
(0.706) \\
\end{array}$ & $\begin{array}{c}-1.428^{* *} \\
(0.676)\end{array}$ & $\begin{array}{c}0.196 \\
(0.870)\end{array}$ & $\begin{array}{l}-0.850 \\
(0.520) \\
\end{array}$ & $\begin{array}{l}-0.388 \\
(0.616) \\
\end{array}$ & $\begin{array}{c}0.159 \\
(0.552) \\
\end{array}$ & $\begin{array}{c}0.124 \\
(0.603)\end{array}$ & $\begin{array}{c}-1.640^{* *} \\
(0.759)\end{array}$ & $\begin{array}{l}-0.269 \\
(0.668) \\
\end{array}$ \\
\hline$M 1_{\text {loc }}-M 1_{E A}(<A)$ & $\begin{array}{c}0.128 \\
(0.564)\end{array}$ & $\begin{array}{l}1.314^{* *} \\
(0.634)\end{array}$ & $\begin{array}{l}0.515 \\
(0.593) \\
\end{array}$ & $\begin{array}{c}0.139 \\
(0.668)\end{array}$ & $\begin{array}{l}0.643 \\
(0.782) \\
\end{array}$ & $\begin{array}{l}-0.685 \\
(1.178) \\
\end{array}$ & $\begin{array}{l}0.695 \\
(0.818) \\
\end{array}$ & $\begin{array}{l}-0.331 \\
(0.616)\end{array}$ & $\begin{array}{c}0.544 \\
(0.668)\end{array}$ \\
\hline$G D P_{l o c}-G D P_{E A}(>A)$ & $\begin{array}{c}-5.553^{* * *} \\
(1.087) \\
\end{array}$ & $\begin{array}{c}-3.589 * * \\
(1.545)\end{array}$ & $\begin{array}{c}-2.694^{*} \\
(1.495) \\
\end{array}$ & $\begin{array}{c}-3.892^{* * *} \\
(1.163) \\
\end{array}$ & $\begin{array}{l}-2.010 \\
(1.404) \\
\end{array}$ & $\begin{array}{l}-1.410 \\
(1.012) \\
\end{array}$ & $\begin{array}{l}-0.869 \\
(1.038) \\
\end{array}$ & $\begin{array}{l}-2.736 \\
(1.695) \\
\end{array}$ & $\begin{array}{r}-1.279 \\
(1.277) \\
\end{array}$ \\
\hline$G D P_{l o c}-G D P_{E A}(<A)$ & $\begin{array}{c}1.461 \\
(1.080)\end{array}$ & $\begin{array}{c}-2.454^{* * *} \\
(0.880)\end{array}$ & $\begin{array}{c}-1.943^{*} \\
(1.152) \\
\end{array}$ & $\begin{array}{c}-2.915^{* * *} \\
(1.036)\end{array}$ & $\begin{array}{c}-2.748^{* *} \\
(1.127)\end{array}$ & $\begin{array}{c}-5.458^{* * *} \\
(1.583) \\
\end{array}$ & $\begin{array}{c}-4.933^{* * *} \\
(1.161)\end{array}$ & $\begin{array}{c}-1.595^{*} \\
(0.844) \\
\end{array}$ & $\begin{array}{c}-3.628^{* * *} \\
(1.016)\end{array}$ \\
\hline$I R_{\text {loc }}-I R_{E A}(>A)$ & $\mid \begin{array}{c}-0.044^{* * *} \\
(0.013)\end{array}$ & $\begin{array}{l}-0.013 \\
(0.017)\end{array}$ & $\begin{array}{l}-0.009 \\
(0.020)\end{array}$ & $\begin{array}{l}-0.017 \\
(0.014)\end{array}$ & $\begin{array}{l}0.008 \\
(0.018)\end{array}$ & $\begin{array}{l}0.008 \\
(0.014)\end{array}$ & $\begin{array}{l}0.007 \\
(0.013)\end{array}$ & $\begin{array}{c}0.014 \\
(0.015)\end{array}$ & $\begin{array}{l}0.007 \\
(0.020)\end{array}$ \\
\hline$I R_{\text {loc }}-I R_{E A}(<A)$ & $\begin{array}{l}-0.010 \\
(0.015) \\
\end{array}$ & $\begin{array}{l}0.005 \\
(0.013) \\
\end{array}$ & $\begin{array}{l}-0.004 \\
(0.020) \\
\end{array}$ & $\begin{array}{l}-0.015 \\
(0.012) \\
\end{array}$ & $\begin{array}{l}-0.001 \\
(0.016) \\
\end{array}$ & $\begin{array}{c}-0.038^{* *} \\
(0.018) \\
\end{array}$ & $\begin{array}{c}-0.034^{*} \\
(0.019) \\
\end{array}$ & $\begin{array}{l}-0.001 \\
(0.017) \\
\end{array}$ & $\begin{array}{l}-0.022 \\
(0.016) \\
\end{array}$ \\
\hline$\pi_{\text {loc }}-\pi_{E A}(>A)$ & $\begin{array}{l}0.004 \\
(0.003)\end{array}$ & $\begin{array}{l}0.007^{*} \\
(0.003)\end{array}$ & $\begin{array}{l}0.008^{*} \\
(0.004)\end{array}$ & $\begin{array}{l}0.001 \\
(0.003)\end{array}$ & $\begin{array}{c}0.004 \\
(0.004)\end{array}$ & $\begin{array}{l}0.003 \\
(0.005)\end{array}$ & $\begin{array}{c}0.004 \\
(0.004)\end{array}$ & $\begin{array}{l}0.000 \\
(0.004)\end{array}$ & $\begin{array}{l}0.003 \\
(0.005)\end{array}$ \\
\hline$\pi_{\text {loc }}-\pi_{E A}(<A)$ & $\begin{array}{l}-0.002 \\
(0.004) \\
\end{array}$ & $\begin{array}{l}0.008^{* *} \\
(0.003) \\
\end{array}$ & $\begin{array}{l}0.009^{*} \\
(0.005) \\
\end{array}$ & $\begin{array}{c}0.003 \\
(0.004) \\
\end{array}$ & $\begin{array}{l}0.009^{* *} \\
(0.004) \\
\end{array}$ & $\begin{array}{l}0.006^{*} \\
(0.003) \\
\end{array}$ & $\begin{array}{c}0.006 \\
(0.004) \\
\end{array}$ & $\begin{array}{c}0.004 \\
(0.003) \\
\end{array}$ & $\begin{array}{c}0.006 \\
(0.004) \\
\end{array}$ \\
\hline$C A(>A)$ & \begin{tabular}{|c|}
$-0.034^{* *}$ \\
$(0.016)$ \\
\end{tabular} & $\begin{array}{l}-0.031 \\
(0.024) \\
\end{array}$ & $\begin{array}{l}-0.042 \\
(0.031) \\
\end{array}$ & $\begin{array}{c}-0.063^{* * *} \\
(0.021) \\
\end{array}$ & $\begin{array}{c}-0.069 * * * \\
(0.026) \\
\end{array}$ & \begin{tabular}{|c|}
$-0.143^{* * *}$ \\
$(0.029)$ \\
\end{tabular} & $\begin{array}{c}-0.146^{* * *} \\
(0.030)\end{array}$ & $\begin{array}{c}-0.125^{* * *} \\
(0.030) \\
\end{array}$ & $\begin{array}{c}-0.126^{* * *} \\
(0.037)\end{array}$ \\
\hline$C A(<A)$ & $\left|\begin{array}{c}-0.204^{* * *} \\
(0.033)\end{array}\right|$ & $\begin{array}{c}-0.097^{* * *} \\
(0.018)\end{array}$ & $\begin{array}{c}-0.088^{* * *} \\
(0.022)\end{array}$ & $\begin{array}{c}-0.057^{* * *} \\
(0.021)\end{array}$ & $\begin{array}{c}-0.117^{* * *} \\
(0.027)\end{array}$ & $\begin{array}{c}-0.053^{* * *} \\
(0.019)\end{array}$ & $\begin{array}{c}-0.074^{* * *} \\
(0.017)\end{array}$ & $\begin{array}{c}-0.053^{* * *} \\
(0.019)\end{array}$ & $\begin{array}{c}-0.080^{* * *} \\
(0.018)\end{array}$ \\
\hline Constant & $\begin{array}{l}-0.664 \\
(1.442) \\
\end{array}$ & $\begin{array}{l}-1.454 \\
(1.408) \\
\end{array}$ & $\begin{array}{l}-1.612 \\
(1.897) \\
\end{array}$ & $\begin{array}{c}-3.515^{* * *} \\
(1.306)\end{array}$ & $\begin{array}{c}-3.096^{* *} \\
(1.501)\end{array}$ & $\begin{array}{l}-1.584 \\
(1.590) \\
\end{array}$ & $\begin{array}{c}-2.793^{*} \\
(1.556) \\
\end{array}$ & $\begin{array}{l}-1.070 \\
(1.694) \\
\end{array}$ & $\begin{array}{c}-2.725^{*} \\
(1.549)\end{array}$ \\
\hline Random effects & Country & Country & Country & Country & Country & Country & Country & Country & Country \\
\hline LRL & 56.91 & 45.39 & 43.99 & 54.77 & 37.75 & 43.55 & 42.35 & 44.75 & 39.38 \\
\hline Wald Test $\left(\chi^{2}\right)$ & 377.3 & 273.9 & 262.6 & 355.8 & 219.5 & 260.9 & 252.2 & 270.3 & 231.6 \\
\hline LR Test $\left(\bar{\chi}^{2}\right)$ & 398.8 & 397.2 & 402.6 & 409.5 & 384.5 & 383.1 & 387.4 & 365.7 & 364.8 \\
\hline Time dummies & YES & YES & YES & YES & YES & YES & YES & YES & YES \\
\hline $\begin{array}{l}\text { Number } \\
\text { of observations }\end{array}$ & 106 & 106 & 106 & 106 & 106 & 106 & 106 & 106 & 106 \\
\hline $\begin{array}{l}\text { Number } \\
\text { of countries }\end{array}$ & 10 & 10 & 10 & 10 & 10 & 10 & 10 & 10 & 10 \\
\hline
\end{tabular}

Note: Standard errors in parentheses. ${ }^{* *} p<0.01,{ }^{* *} p<0.05,{ }^{*} p<0.1$. $>$ A denotes the cases when frequency of searching in events is above the average, while $<A$ denotes the cases when frequency of searching in events is below the average.

Source: own calculation 
Table 5 | Robustness of Augmented Portfolio Balance Model: Negative Changes of Foreign Currency Deposits' Ratio

\begin{tabular}{|c|c|c|c|c|c|c|c|c|c|}
\hline & \multicolumn{9}{|c|}{ Dependent variable: $\Delta$ of foreign currency deposits to total deposits $(\operatorname{In})(\Delta<0)$} \\
\hline & \multicolumn{9}{|c|}{ Google keywords } \\
\hline & $\begin{array}{l}\text { Currency } \\
\text { code }\end{array}$ & "Debt" & “Money" & "Growth" & $\begin{array}{c}\text { "Employ- } \\
\text { ment" }\end{array}$ & "Political" & $\begin{array}{l}\text { "Parlia- } \\
\text { ment" }\end{array}$ & "Premier" & “Risk” \\
\hline $\begin{array}{l}\text { Independent } \\
\text { variables }\end{array}$ & (10) & (11) & (12) & (13) & (14) & (15) & (16) & (17) & (18) \\
\hline $\operatorname{NEER}(>\mathrm{A})$ & $\begin{array}{c}-0.793^{* * *} \\
(0.253)\end{array}$ & $\begin{array}{l}-0.227 \\
(0.256)\end{array}$ & $\begin{array}{l}-0.335 \\
(0.242)\end{array}$ & $\begin{array}{l}-0.228 \\
(0.211)\end{array}$ & $\begin{array}{l}-0.083 \\
(0.257)\end{array}$ & $\begin{array}{l}-0.433^{*} \\
(0.235)\end{array}$ & $\begin{array}{l}-0.278 \\
(0.235)\end{array}$ & $\begin{array}{l}-0.211 \\
(0.265)\end{array}$ & $\begin{array}{l}-0.380 \\
(0.258)\end{array}$ \\
\hline $\operatorname{NEER}(<\mathrm{A})$ & $\begin{array}{c}-0.705^{* * *} \\
(0.239)\end{array}$ & $\begin{array}{l}-0.202 \\
(0.246)\end{array}$ & $\begin{array}{l}-0.318 \\
(0.230)\end{array}$ & $\begin{array}{l}-0.212 \\
(0.217)\end{array}$ & $\begin{array}{l}-0.065 \\
(0.264)\end{array}$ & $\begin{array}{c}-0.482^{* *} \\
(0.241)\end{array}$ & $\begin{array}{l}-0.294 \\
(0.238)\end{array}$ & $\begin{array}{l}-0.349 \\
(0.262)\end{array}$ & $\begin{array}{l}-0.422 \\
(0.261)\end{array}$ \\
\hline$M 1_{\text {loc }}-M 1_{E A}(>A)$ & $\begin{array}{c}0.015 \\
(0.706) \\
\end{array}$ & $\begin{array}{c}0.241 \\
(0.649) \\
\end{array}$ & $\begin{array}{c}0.047 \\
(0.519) \\
\end{array}$ & $\begin{array}{l}-0.011 \\
(0.668) \\
\end{array}$ & $\begin{array}{l}1.426^{* *} \\
(0.618)\end{array}$ & $\begin{array}{c}0.586 \\
(0.576) \\
\end{array}$ & $\begin{array}{c}0.870 \\
(0.540) \\
\end{array}$ & $\begin{array}{l}0.060 \\
(0.629) \\
\end{array}$ & $\begin{array}{l}0.965 \\
(0.610) \\
\end{array}$ \\
\hline$M 1_{\text {loc }}-M 1_{E A}(<A)$ & $\begin{array}{c}0.654 \\
(0.606)\end{array}$ & $\begin{array}{l}1.138^{*} \\
(0.651)\end{array}$ & $\begin{array}{l}2.967^{* * *} \\
(0.726)\end{array}$ & $\begin{array}{l}-0.263 \\
(0.563)\end{array}$ & $\begin{array}{c}0.036 \\
(0.803)\end{array}$ & $\begin{array}{c}1.083 \\
(0.734)\end{array}$ & $\begin{array}{l}0.301 \\
(0.746)\end{array}$ & $\begin{array}{l}0.977^{*} \\
(0.585)\end{array}$ & $\begin{array}{l}0.710 \\
(0.732)\end{array}$ \\
\hline$G D P_{l o c}-G D P_{E A}(>A)$ & $\begin{array}{l}-1.809 \\
(1.150) \\
\end{array}$ & $\begin{array}{c}2.154 \\
(1.601)\end{array}$ & $\begin{array}{l}-0.575 \\
(1.123) \\
\end{array}$ & $\begin{array}{l}-2.078^{*} \\
(1.230) \\
\end{array}$ & $\begin{array}{l}-1.806 \\
(1.366) \\
\end{array}$ & $\begin{array}{l}-1.584 \\
(1.258) \\
\end{array}$ & $\begin{array}{l}-2.826^{*} \\
(1.444)\end{array}$ & $\begin{array}{c}-3.078^{* *} \\
(1.315)\end{array}$ & $\begin{array}{c}-3.252^{* *} \\
(1.388)\end{array}$ \\
\hline$G D P_{l o c}-G D P_{E A}(<A)$ & $\begin{array}{l}-2.473^{* *} \\
(1.238)\end{array}$ & $\begin{array}{c}-4.100^{* * *} \\
(1.112)\end{array}$ & $\begin{array}{c}-5.607^{* * *} \\
(1.220)\end{array}$ & $\begin{array}{l}-1.730^{*} \\
(1.007)\end{array}$ & $\begin{array}{c}-3.410^{* * *} \\
(1.275)\end{array}$ & $\begin{array}{l}-2.203^{*} \\
(1.169)\end{array}$ & $\begin{array}{l}-0.985 \\
(1.223)\end{array}$ & $\begin{array}{l}-0.969 \\
(1.140)\end{array}$ & $\begin{array}{l}-2.000^{*} \\
(1.150)\end{array}$ \\
\hline$I R_{l o c}-I R_{E A}(>A)$ & $\begin{array}{c}-0.043^{* * *} \\
(0.013)\end{array}$ & $\begin{array}{l}-0.016 \\
(0.013) \\
\end{array}$ & $\begin{array}{c}-0.033^{* *} \\
(0.015)\end{array}$ & $\begin{array}{l}0.017^{*} \\
(0.010)\end{array}$ & $\begin{array}{l}-0.004 \\
(0.014) \\
\end{array}$ & $\begin{array}{c}0.011 \\
(0.011) \\
\end{array}$ & $\begin{array}{l}0.007 \\
(0.011)\end{array}$ & $\begin{array}{l}0.007 \\
(0.011) \\
\end{array}$ & $\begin{array}{l}0.008 \\
(0.013) \\
\end{array}$ \\
\hline$I R_{l o c}-I R_{E A}(<A)$ & $\begin{array}{l}0.017 \\
(0.011)\end{array}$ & $\begin{array}{l}0.010 \\
(0.013)\end{array}$ & $\begin{array}{l}0.024^{*} \\
(0.012)\end{array}$ & $\begin{array}{l}0.029^{* *} \\
(0.012)\end{array}$ & $\begin{array}{l}0.009 \\
(0.013)\end{array}$ & $\begin{array}{c}-0.066^{* * *} \\
(0.017)\end{array}$ & $\begin{array}{c}-0.059^{* * *} \\
(0.019)\end{array}$ & $\begin{array}{l}-0.013 \\
(0.014)\end{array}$ & $\begin{array}{l}-0.014 \\
(0.013)\end{array}$ \\
\hline$\pi_{l o c}-\pi_{E A}(>A)$ & $\begin{array}{l}0.011^{* *} \\
(0.003) \\
\end{array}$ & $\begin{array}{l}0.010^{* * *} \\
(0.003)\end{array}$ & $\begin{array}{l}0.015^{* * *} \\
(0.003)\end{array}$ & $\begin{array}{c}0.003 \\
(0.003) \\
\end{array}$ & $\begin{array}{l}0.014^{* * *} \\
(0.004)\end{array}$ & $\begin{array}{l}0.007^{* *} \\
(0.003)\end{array}$ & $\begin{array}{l}0.011^{*+*} \\
(0.004)\end{array}$ & $\begin{array}{l}0.009^{* * *} \\
(0.003)\end{array}$ & $\begin{array}{l}0.008^{* *} \\
(0.004)\end{array}$ \\
\hline$\pi_{l o c}-\pi_{E A}(<A)$ & $\begin{array}{c}0.003 \\
(0.004)\end{array}$ & $\begin{array}{l}0.011^{* * *} \\
(0.003)\end{array}$ & $\begin{array}{l}0.014^{* * * *} \\
(0.004)\end{array}$ & $\begin{array}{l}-0.004 \\
(0.003)\end{array}$ & $\begin{array}{l}0.010^{* * *} \\
(0.003)\end{array}$ & $\begin{array}{l}0.011^{* * *} \\
(0.003)\end{array}$ & $\begin{array}{l}0.012^{* * *} \\
(0.003)\end{array}$ & $\begin{array}{l}0.014^{* * *} \\
(0.003)\end{array}$ & $\begin{array}{l}0.007^{*} \\
(0.003)\end{array}$ \\
\hline$C A(>A)$ & $\begin{array}{c}-0.092^{* * *} \\
(0.024)\end{array}$ & $\begin{array}{c}-0.069^{* *} \\
(0.028)\end{array}$ & $\begin{array}{c}-0.081^{* * *} \\
(0.027)\end{array}$ & $\begin{array}{c}-0.094^{* * *} \\
(0.025)\end{array}$ & $\begin{array}{c}-0.101^{* * *} \\
(0.030)\end{array}$ & $\begin{array}{c}-0.132^{* * *} \\
(0.026)\end{array}$ & $\begin{array}{c}-0.119^{* * *} \\
(0.024)\end{array}$ & $\begin{array}{c}-0.161^{* * *} \\
(0.027)\end{array}$ & $\begin{array}{c}-0.156^{* * *} \\
(0.033)\end{array}$ \\
\hline$C A(<A)$ & $\begin{array}{c}-0.177^{* * *} \\
(0.030)\end{array}$ & $\begin{array}{c}-0.102^{* * *} \\
(0.028)\end{array}$ & $\begin{array}{c}-0.103^{* * *} \\
(0.024)\end{array}$ & $\begin{array}{c}-0.054^{* *} \\
(0.025)\end{array}$ & $\begin{array}{c}-0.098^{* * *} \\
(0.029)\end{array}$ & $\begin{array}{c}-0.075^{* * *} \\
(0.029)\end{array}$ & $\begin{array}{c}-0.068^{* *} \\
(0.031)\end{array}$ & $\begin{array}{l}-0.037 \\
(0.025)\end{array}$ & $\begin{array}{c}-0.101^{* * *} \\
(0.027)\end{array}$ \\
\hline Constant & $\begin{array}{c}1.508 \\
(1.317)\end{array}$ & $\begin{array}{l}-1.336 \\
(1.318)\end{array}$ & $\begin{array}{l}-0.795 \\
(1.249)\end{array}$ & $\begin{array}{l}-0.990 \\
(1.190)\end{array}$ & $\begin{array}{l}-1.815 \\
(1.374)\end{array}$ & $\begin{array}{l}-0.035 \\
(1.266)\end{array}$ & $\begin{array}{l}-0.712 \\
(1.266)\end{array}$ & $\begin{array}{l}-0.929 \\
(1.362)\end{array}$ & $\begin{array}{l}-0.121 \\
(1.372)\end{array}$ \\
\hline Random effects & Country & Country & Country & Country & Country & Country & Country & Country & Country \\
\hline LRL & 57.80 & 50.66 & 59.42 & 67.60 & 45.20 & 54.06 & 52.21 & 58.86 & 46.41 \\
\hline Wald Test $\left(\chi^{2}\right)$ & 255.5 & 211.4 & 263.0 & 322.9 & 182.2 & 232.1 & 220.7 & 260.8 & 189.8 \\
\hline LR Test $\left(\bar{\chi}^{2}\right)$ & 418.1 & 436.0 & 439.4 & 469.2 & 420.3 & 442.5 & 426.4 & 438.7 & 407.2 \\
\hline Time dummies & YES & YES & YES & YES & YES & YES & YES & YES & YES \\
\hline $\begin{array}{l}\text { Number } \\
\text { of observations }\end{array}$ & 128 & 128 & 128 & 128 & 128 & 128 & 128 & 128 & 128 \\
\hline $\begin{array}{l}\text { Number } \\
\text { of countries }\end{array}$ & 10 & 10 & 10 & 10 & 10 & 10 & 10 & 10 & 10 \\
\hline
\end{tabular}

Note: Standard errors in parentheses. ${ }^{* * *} p<0.01,{ }^{* *} p<0.05,{ }^{*} p<0.1$. $>$ A denotes the cases when frequency of searching in events is above the average, while $<$ A denotes the cases when frequency of searching in events is below the average.

Source: own calculation 


\section{Conclusions}

The paper investigates the impact of behavioural attention on the households' foreign currency savings as response to the external macroeconomic shocks. We apply two leading macroeconomic models based on competing macroeconomic approaches to exchange rate: Monetary Model, and Portfolio Balance Model. We have also extended the macroeconomic models by including individuals' attention to the specific risks and search interest in specific keywords on Google in order to assess the impact of information's communication channel on the aggregate households' foreign currency savings.

The empirical estimations provide substantial evidence that macroeconomic determinants derived from the basic and modified Portfolio Balance Models are significant in explaining aggregate households' foreign currency savings. The empirical findings suggest that exchange rate movements have a significant impact on the share of foreign currency deposits, while the appreciation of the national currency affects negatively returns on foreign currency deposits and causes reallocation in favour on deposits in national currency, and conversely. The empirical findings also demonstrate that a more intense domestic economic growth motivates households to increase the share of deposits in national currency suggesting about increased confidence in national currency related with the improving economic situation in country. In summary, aggregate households' foreign currency savings are affected mostly by exchange rates movements and economic shocks, while the effect of inflation and interest rate shocks is very limited.

We can assume that households' reaction to the external macroeconomic shocks (exchange rates and economic shocks) could be affected by their search activities on Google. Thus, we can summarize that aggregate households' foreign currency savings could be influenced by individuals more intense attention in specific risks and higher search interest on Google for specific keywords. In other words, households are more sensitive to macroeconomic factors when they search for information on Google about these factors or related risks. In addition, we assume that increased households' attention to the specific risks and more intense search interest on Google plays a crucial role in the portfolio rebalancing.

Our findings could be used for policy makers' communication strategy, especially in the environment of very low interest rates. The empirical findings suggest that the communication strategy (including the communication channels) of the central banks and governments during financial turmoil and economic downturn periods could affect the households' foreign currency savings. 


\section{Appendices}

\section{Appendix 1 | Definition of Variables}

\begin{tabular}{|c|c|c|}
\hline Variable & Definition & Source \\
\hline SAVE & $\begin{array}{l}\text { Households' deposits in foreign currency } \\
\text { to total households' deposits held at } \\
\text { the monetary financial institutions (MFIs) } \\
\text { ratio }\end{array}$ & $\begin{array}{l}\text { Bank of Estonia, Bank of Lithuania, Bank } \\
\text { of Latvia, Czech National Bank, Bulgarian } \\
\text { National Bank, National Bank of Poland, } \\
\text { National Bank of Hungary, Swedish } \\
\text { National Bank, National Bank of Romania, } \\
\text { Bank of England }\end{array}$ \\
\hline NEER & $\begin{array}{l}\text { Nominal effective exchange rate as } \\
\text { the weighted average of bilateral nominal } \\
\text { exchange rates against the currencies } \\
\text { of selected } 42 \text { trading partners } \\
\text { (chain index) }\end{array}$ & Eurostat \\
\hline IR & $\begin{array}{l}\text { Short-term ( } 3 \text { months) money market } \\
\text { interest rate }(\%)\end{array}$ & $\begin{array}{l}\text { Eurostat, OECD (Main Economic Indicators), } \\
\text { IMF (International Financial Statistics), } \\
\text { Czech National Bank }\end{array}$ \\
\hline$G D P$ & $\begin{array}{l}\text { Gross domestic product in current prices } \\
\text { (chain index) }\end{array}$ & Eurostat \\
\hline$M$ & $\begin{array}{l}\text { Money supply M1 (currency } \\
\text { in circulation and demand deposits) } \\
\text { (chain index) }\end{array}$ & $\begin{array}{l}\text { IMF (International Financial Statistics), } \\
\text { OECD (Main Economic Indicators) }\end{array}$ \\
\hline$C A$ & Current account balance (chain index) & Eurostat, Bulgarian National Bank \\
\hline REM & Workers' remittances (chain index) & IMF (Balance of Payments) \\
\hline$\pi$ & Inflation measured by GDP deflator (\%) & Eurostat \\
\hline $\begin{array}{l}\text { Google } \\
\text { keywords }\end{array}$ & $\begin{array}{l}\text { Google Trends keywords in national } \\
\text { languages reflecting different risks: } \\
\text { geopolitical risk ("attack", "war", } \\
\text { "freedom"), political risk ("political", } \\
\text { "corruption", "election", "president", } \\
\text { "parliament", "senate", "premier"), financial } \\
\text { risk ("money", "bank", "risk", "loss"), } \\
\text { currency risk (currency code, currency } \\
\text { name), unemployment risk ("employment", } \\
\text { "unemployment", "job"), credit risk } \\
\text { ("debt", "indebtedness"), and general } \\
\text { economic situation ("inflation", "growth", } \\
\text { "competitiveness", "recession", "crisis") }\end{array}$ & Google Trends database \\
\hline
\end{tabular}

Note: Data on GDP and M1 are seasonally adjusted.

Source: own processing 
Appendix 2 | Google Keywords

\begin{tabular}{|c|c|}
\hline Google keyword & Translation to the local languages \\
\hline Currency code & CZK, PLN, HUF, LTL, GBP, BGN, SEK, RON, EEK, LVL \\
\hline Currency name & koruna, złoty, forint, litas, pound, лев, krona, leu, kroon, lats \\
\hline "Crisis" & krize, kryzys, válság, krizè, crisis, криза, kris, criză, kriis, krīze \\
\hline "Inflation" & $\begin{array}{l}\text { inflace, inflacja, infláció, infliacija, inflation, инфлация, inflation, umflare, inflatsioon, } \\
\text { inflācija }\end{array}$ \\
\hline "Debt" & dluh, dług, adósság, skola, debt, дълг, skuld, datorie, võlg, parāds \\
\hline “Indebtedness" & $\begin{array}{l}\text { zadlužení, zadłużenie, eladósodottság, isiskolinimas, indebtedness, задълженост, } \\
\text { skuldsättning, îndatorare, võlgnevus, parādsaistības }\end{array}$ \\
\hline "Money" & peníze, pieniądze, pénz, pinigai, money, пари, pengar, bani, raha, nauda \\
\hline "Bank" & $\begin{array}{l}\text { banka, bankovní, banky, banku, bankowy, banki, bank, banki, bank, bankas, } \\
\text { bankininkystè, bankai, bank, banking, banks, банкови, банково, дело, банки, bank, } \\
\text { bank, banker, bancare, servicii, bancare, băncile, pank, pangandus, pankade, banku, } \\
\text { banku, bankas }\end{array}$ \\
\hline "Growth" & růst, wzrost, növekedés, augimas, growth, растеж, tillväxt, creștere, kasv, pieaugums \\
\hline "Employment" & $\begin{array}{l}\text { zaměstnanost, zatrudnienie, foglalkoztatás, užimtumas, employment, наемане на } \\
\text { работа, sysselsättning, ocuparea, forței de muncă, tööhõive, nodarbinātība }\end{array}$ \\
\hline "Unemployment" & $\begin{array}{l}\text { nezaměstnanost, bezrobocie, munkanélküliség, nedarbas, unemployment, } \\
\text { безработица, arbetslöshet, șomaj, tööpuudus, bezdarbs }\end{array}$ \\
\hline “Job" & práce, praca, munka, darbas, job, работа, arbete, lucru, töö, darbs \\
\hline "Competitiveness" & $\begin{array}{l}\text { konkurenceschopnost, konkurencyjność, versenyképesség, Konkurencingumas, } \\
\text { competitiveness, конкурентоспособност, konkurrenskraft, competitivitate, } \\
\text { konkurentsivõime, konkurētspēja }\end{array}$ \\
\hline "Recession" & $\begin{array}{l}\text { recese, recesja, recesszió, nuosmukis, recession, спад, recession, recesiune, langus, } \\
\text { lejupslīde }\end{array}$ \\
\hline "Attack" & útok, atak, támadás, ataka, attack, атака, attack, atac, rünnak, uzbrukums \\
\hline "War" & válka, wojna, háború, karas, war, война, krig, război, sõda, karš \\
\hline "Corruption" & $\begin{array}{l}\text { korupce, korupcja, vesztegetés, korupcija, corruption, корупция, korruption, } \\
\text { corupție, korruptsioon, korupcija }\end{array}$ \\
\hline "Political" & $\begin{array}{l}\text { politický, politická, politika, polityczne, polityczne, polityka, politikai, politikai, } \\
\text { politika, politinė, politinė, Politika, political, political, politics, политическа, } \\
\text { политическа, политика, politiskt, politiskt, politik, politic, politic, politica, poliitilise, } \\
\text { poliitilise, poliitika, politiskā, politiskā, politika }\end{array}$ \\
\hline "Election" & $\begin{array}{l}\text { volby, wyborczy, választási, rinkimai, election, избори, val, alegere, valimised, } \\
\text { vēlēšanas }\end{array}$ \\
\hline "Freedom" & $\begin{array}{l}\text { svoboda, wolność, szabadság, laisvè, freedom, свобода, frihet, libertate, vabadus, } \\
\text { brīvība }\end{array}$ \\
\hline "President" & $\begin{array}{l}\text { prezident, prezes, elnök, prezidentas, president, президент, president, Președintele, } \\
\text { president, prezidents }\end{array}$ \\
\hline "Parliament" & $\begin{array}{l}\text { parlament, parlament, parlament, parlamentas, parliament, парламент, parlament, } \\
\text { parlament, parlament, parlaments }\end{array}$ \\
\hline "Senate" & senát, senat, szenátus, senatas, senate, сенат, senat, senat, senat, senāts \\
\hline "Premier" & $\begin{array}{l}\text { premiér, premier, miniszterelnök, pirmaeilis, premier, премиер, premier, premier, } \\
\text { peaminister, pirmais }\end{array}$ \\
\hline "Risk" & riziko, ryzyko, kockázat, rizika, risk, puck, risk, risc, risk, risks \\
\hline “Loss” & ztráta, strata, veszteség, praradimas, loss, загуба, förlust, pierdere, kaotus, zaudējums \\
\hline
\end{tabular}

Source: own processing 


\section{Appendix 3 | Methodology of Bayesian Model Averaging}

Google keywords are specific type of the data called as Fat Data where the number of variables is large. Moreover, we have no appropriate theoretical background to reduce uncertainty in the model selection. Therefore, we follow Bayesian Model Averaging approach, special framework provided by De Luca and Magnus (2011) where a regression model is defined by the form:

$$
y_{i t}=X_{1 i t} \beta_{1}+X_{2 i t} \beta_{2 c t}+u_{i t}
$$

where $y_{i t}$ represents a share of deposits in foreign currency in a country $i=1, \ldots, n$ and time $t$. We assume that $k_{1} \geq 1, k_{2} \geq 0, \mathrm{k}=k_{1}+k_{2} \leq n-1$ and the design matrix $\mathbf{X}=\left(\mathrm{X}_{1}, \mathrm{X}_{2}\right)$ has full column-rank $k$. The deterministic regressors $X_{j}$ represents $n \times k_{j}$ matrices of observations on two subsets of regressors $X_{1}$ and $X_{2} . \beta_{1}$ and $\beta_{2}$ are unknown regression parameters and $u \sim N\left(0, \sigma^{2}\right)$. The first subset of regressors $X_{1}$ contains macroeconomic determinants of the Portfolio Balance Model introduced by Hooper and Morton (1982) and $X_{2}$ contains frequencies of selected keyword searches (auxiliary variables) to which model uncertainty is confined.

There are $2^{k_{2}}$ possible models, thus, there are $2^{k_{2}}$ possible choices for $X_{2}$. We consider up to 26 regressors to be included in the model that means $2^{26}$, that is $67,108,864$ individual models. A model-averaging estimate of $\beta_{1}$ is given by:

$$
\hat{\beta}_{1}=\sum_{i=1}^{I} \lambda_{i} \hat{\beta}_{1 i}
$$

Where $\lambda_{i}$ represent non-negative random weights which reflect our confidence in model $M_{i}$ based on prior beliefs. We combine prior beliefs on the unknown elements of the model with the additional information coming from the data. We employed estimator developed by Magnus et al. (2010) based on the distinction between focus and auxiliary regressors. Prior beliefs are given by non-informative priors on the focus parameters $\beta_{1}$, the error variance $\sigma^{2}$, and informative Gaussian prior on the auxiliary parameters $\beta_{2 i}$. The conditional posterior distribution $p\left(\beta_{1}, \beta_{2 i}, \sigma^{2} \mid y, M_{i}\right)$ is given by sample likelihood function:

$$
\mathrm{p}\left(\mathrm{y} \mid \beta_{1}, \beta_{2 i}, \sigma^{2}, M_{i}\right) \infty\left(\sigma^{2}\right)^{-n / 2} \exp \left(-\frac{\varepsilon_{i}{ }^{\tau} \varepsilon_{i}}{2 \sigma^{2}}\right)
$$

with the conditional joint prior distribution:

$$
\mathrm{p}\left(\beta_{1}, \beta_{2}, \sigma^{2} \mid M_{i}\right) \infty\left(\sigma^{2}\right)^{\left(k_{2 i}+2\right) / 2} \exp \left(-\frac{\beta_{2 i}{ }^{\tau} V_{0 i}^{-1} \beta_{2 i}}{2 \sigma^{2}}\right)
$$

where $V_{0 i}^{-1}=g X_{2 i} M_{1} X_{2 i}$ was proposed by Zellner (1986) and Fernández et al. (2001) and $g=1 / \max \left(n, k_{2}^{2}\right)$ is a constant scalar for each model $M_{i}$. Then we obtain results for every model under consideration and average them where the weights in the averaging are the posterior model probabilities:

$$
\lambda_{i}=p\left(M_{i} \mid \mathrm{y}\right)=\frac{p\left(M_{i}\right) p\left(y \mid M_{i}\right)}{\sum_{j=1}^{I} p\left(M_{j}\right) p\left(y \mid M_{j}\right)}
$$


where $p\left(M_{i}\right)$ is the prior probability of model $M_{i}$ and $p\left(y \mid M_{i}\right)$ is the marginal likelihood of $y$ given model $M_{i}$. The unconditional estimates of $\beta_{1}$ and $\beta_{2}$ are computed as:

and

$$
\hat{\beta}_{1}=E\left(\beta_{1} \mid \mathrm{y}\right)=\sum_{i=1}^{I} \lambda_{i} \hat{\beta}_{1 i}
$$

$$
\hat{\beta}_{2}=E\left(\beta_{2} \mid \mathrm{y}\right)=\sum_{i=1}^{I} \lambda_{i} T_{i} \hat{\beta}_{2 i}
$$

where the $T_{i}$ are $k_{2} \times k_{2 i}$ matrices defined by $T_{i}^{\tau}=\left(I_{k_{2 i}}, 0\right)$, that transform the conditional esimates $\hat{\beta}_{2 i}$ in $k_{2} \mathrm{x} 1$ vectors by setting to zero the elements of $\beta_{2}$, which are excluded from model $M_{i}$. 
Appendix 4 | Bayesian Model Averaging Results (foreign currency deposits to total deposits (In) as a dependent variable (2004-2014))

\begin{tabular}{|c|c|c|c|c|c|c|}
\hline & \multicolumn{3}{|c|}{$\begin{array}{c}\text { Basic models with non-lagged } \\
\text { regressors }\end{array}$} & \multicolumn{3}{|c|}{$\begin{array}{c}\text { Basic models with lagged } \\
\text { regressors }\end{array}$} \\
\hline & $\begin{array}{l}\text { Posterior } \\
\text { Inclusion } \\
\text { Probability }\end{array}$ & $\begin{array}{l}\text { Posterior } \\
\text { Mean }\end{array}$ & $\begin{array}{c}\text { Posterior } \\
\text { Standard } \\
\text { Error }\end{array}$ & $\begin{array}{l}\text { Posterior } \\
\text { Inclusion } \\
\text { Probability }\end{array}$ & $\begin{array}{l}\text { Posterior } \\
\text { Mean }\end{array}$ & $\begin{array}{c}\text { Posterior } \\
\text { Standard } \\
\text { Error }\end{array}$ \\
\hline Time dummies & \multicolumn{3}{|c|}{ Yes } & \multicolumn{3}{|c|}{ Yes } \\
\hline Constant & 1.00 & -6.6037 & 3.8413 & 1.00 & -5.9503 & 3.5369 \\
\hline NEER & 1.00 & -0.2152 & 0.6774 & 1.00 & -0.3535 & 0.6109 \\
\hline$\pi_{\text {local }}-\pi_{E A}$ & 1.00 & 0.0589 & 0.0080 & 1.00 & 0.0565 & 0.0076 \\
\hline$G D P_{\text {local }}-G D P_{E A}$ & 1.00 & -4.9153 & 1.7952 & 1.00 & -5.0017 & 1.8217 \\
\hline CA & 1.00 & 0.0887 & 0.0382 & 1.00 & 0.0886 & 0.0380 \\
\hline Currency code & 1.00 & 0.6176 & 0.1164 & 1.00 & 0.6642 & 0.1402 \\
\hline Currency name & 0.31 & -0.1039 & 0.1765 & 0.55 & -0.2165 & 0.2206 \\
\hline "Crisis" & 0.04 & -0.0012 & 0.0238 & 0.04 & -0.0007 & 0.0230 \\
\hline "Inflation" & 0.08 & -0.0184 & 0.0898 & 0.06 & -0.0117 & 0.0709 \\
\hline "Debt" & 0.99 & -0.5624 & 0.18 & 0.98 & -0.5358 & 0.1733 \\
\hline “Indebtedness" & 0.06 & -0.0044 & 0.0397 & 0.05 & -0.0034 & 0.0370 \\
\hline "Money" & 0.99 & 0.9281 & 0.2647 & 0.99 & 0.9660 & 0.2541 \\
\hline "Bank" & 0.40 & 0.1352 & 0.1892 & 0.29 & 0.0930 & 0.1655 \\
\hline "Growth" & 0.78 & -0.3181 & 0.2064 & 0.87 & -0.3717 & 0.1880 \\
\hline "Employment" & 1.00 & 0.5386 & 0.0973 & 1.00 & 0.5219 & 0.1049 \\
\hline “Unemployment” & 0.06 & 0.0054 & 0.0400 & 0.05 & 0.0024 & 0.0305 \\
\hline "Job" & 0.06 & 0.0135 & 0.1162 & 0.06 & 0.0159 & 0.1162 \\
\hline "Competitiveness" & 0.51 & 0.1879 & 0.2108 & 0.46 & 0.1752 & 0.2130 \\
\hline "Recession" & 0.50 & 0.1312 & 0.1501 & 0.28 & 0.0607 & 0.1136 \\
\hline "Attack" & 0.16 & 0.0261 & 0.0710 & 0.18 & 0.0313 & 0.0777 \\
\hline "War" & 0.05 & 0.0004 & 0.0440 & 0.05 & -0.0006 & 0.0412 \\
\hline "Corruption" & 0.04 & 0.0017 & 0.0212 & 0.04 & 0.0013 & 0.0206 \\
\hline "Political" & 1.00 & 1.2545 & 0.2444 & 1.00 & 1.3677 & 0.2432 \\
\hline "Election" & 0.06 & -0.0023 & 0.0132 & 0.08 & -0.0037 & 0.0164 \\
\hline "Freedom" & 0.10 & 0.0105 & 0.0386 & 0.11 & 0.0115 & 0.0401 \\
\hline "President" & 0.21 & 0.0462 & 0.1036 & 0.31 & 0.0740 & 0.1255 \\
\hline "Parliament" & 0.92 & -0.5364 & 0.2376 & 0.88 & -0.4954 & 0.2450 \\
\hline "Senate" & 0.07 & -0.0071 & 0.0412 & 0.08 & -0.0093 & 0.0458 \\
\hline "Premier" & 1.00 & -0.5180 & 0.1031 & 1.00 & -0.5040 & 0.0969 \\
\hline "Risk" & 0.99 & -0.9834 & 0.2023 & 1.00 & -0.9877 & 0.1984 \\
\hline "Loss" & 0.14 & 0.0360 & 0.1069 & 0.11 & 0.0220 & 0.0813 \\
\hline No. of observations & \multicolumn{3}{|c|}{162} & \multicolumn{3}{|c|}{162} \\
\hline k1 & \multicolumn{3}{|c|}{45} & \multicolumn{3}{|c|}{45} \\
\hline k2 & \multicolumn{3}{|c|}{26} & \multicolumn{3}{|c|}{26} \\
\hline
\end{tabular}

Source: own calculation 


\section{References}

Allen, L., Saunders, A. (2014). Risk Management in Banking. In The Oxford Handbook of Banking. Editors Allen N. Berger, Philip Molyneux and John O.S. Wilson. Oxford University Press, Oxford, p. 1104.

Arifovic, J. (2001). Evolutionary Dynamics of Currency Substitution. Journal of Economic Dynamics and Control, 25(3-4), 395-417, https://doi.org/10.1016/s0165-1889(00)00031-2

Boughton, J. M. (1988). The Monetary Approach to Exchange Rates: What Now Remains? International Finance Section. ISBN 978-0-881-65078-5.

Bresser-Pereira, L. C., Araújo, E., Gala, P. (2014). An Empirical Study of the Substitution of Foreign for Domestic Savings in Brazil. Economia, 15(1), 54-67, https://doi.org/10.1016/j.econ.2014.04.001

Bulut, L. (2015). Google Trends and Forecasting Performance of Exchange Rate Models. IPEK University Department of Economics. Working Paper No. 15-05, https://doi.org/10.2139/ ssrn.2641796

Choi, H., Varian, H. (2012). Predicting the Present with Google Trends. The Economic Record, 88, 2-9, https://doi.org/10.1111/j.1475-4932.2012.00809.x

Da, Z., Engelberg, J., Gao, P. (2011). In Search of Attention. The Journal of Finance, 66, 1461-1499, https://doi.org/10.1111/j.1540-6261.2011.01679.x

De Luca, G., Magnus, J. R. (2011). Bayesian Model Averaging and Weighted-Average Least Squares: Equivariance, Stability, and Numerical Issues. Stata Journal, 11(4), 518-544.

Dornbusch, R. (1976). Expectations and Exchange Rate Dynamics. Journal of Political Economy, 84(6), 1161-1176, https://doi.org/1086/260506

Fernández, C., Ley, E., Steel, M. F. J. (2001). Model Uncertainty in Cross-Country Growth Regressions. Journal of Applied Econometrics, 16(5), 563-576, https://doi.org/10.1002/ jae.623

Frankel, J. (1979). On the Mark: A Theory of Floating Exchange Rates Based on Real Interest Differentials. American Economic Review, 69, 610-622.

Guzi, M., de Pedraza García, P. (2015). A Web Survey Analysis of Subjective Well-Being", International Journal of Manpower, 36(1), 48-67, https://doi.org/10.1108/ ijm-12-2014-0237

Harvey, J. T. (1996). Orthodox Approaches to Exchange Rate Determination:

A Survey. Journal of Post Keynesian Economics, 18, 567-583, https://doi.org/ 10.1080/01603477.1996.11490087

Hooper, P., Morton, J. E. (1982). Fluctuations in the Dollar: A Model of Nominal and Real Exchange Rate Determination. Journal of International Money and Finance, 1, 39-56, https://doi.org/10.1016/0261-5606(82)90004-3

Irresberger, F., Mühlnickel, J., Weiß, G. N. F. (2015). Explaining Bank Stock Performance with Crisis Sentiment. Journal of Banking \& Finance, 59, 311-329, https://doi.org/10.1016/j. jbankfin.2015.06.001

Isard, P. (1978). Exchange Rate Determination: A Survey of Popular Views and Recent Models. Princeton Studies in International Finance, 42.

Kapounek, S., Deltuvaitè, V., Koráb, P. (2016a). Determinants of Foreign Currency Savings: Evidence from Google Search Data. Procedia - Social and Behavioral Sciences, 220, 166-176, https://doi.org/10.1016/j.sbspro.2016.05.481 
Kapounek, S., Korab, P., Deltuvaite, V. (2016b). (Ir)rational Households' Saving Behavior? An Empirical Investigation. Procedia Economics and Finance, 39, 625-633, https://doi. org/10.1016/S2212-5671(16)30309-4

Kenen, P. B. (1985). Macroeconomic Theory and Policy: How the Closed Economy Was Opened. Handbook of International Economics, 2, 625-677, https://doi.org/10.1016/ s1573-4404(85)02004-4

Knoll, M. A. Z. (2010). The Role of Behavioral Economics and Behavioral Decision Making in Americans' Retirement Savings Decisions. Social Security Bulletin, 70(4).

Lee, T. D., Haley, E., Woong, Y. T., Chung, W. (2011). US Retirement Financial Services Advertising's Financial Information Provisions, Communication Strategies and Judgmental Heuristic Cues. Journal of Consumer Affairs, 45(3), 391-418, https://doi. org/10.1111/j.1745-6606.2011.01210.x

Magnus, J. R., Powell, O., Prüfer, O. (2010). A Comparison of Two Model Averaging Techniques with an Application to Growth Empirics. Journal of Econometrics, 154(2), 139-153, https://doi.org/10.1016/j.jeconom.2009.07.004

Mark, N. C. (1995). Exchange Rates and Fundamentals: Evidence on Long Horizon Predictability. American Economic Review, 85, 201-218.

Mussa, M. (1979). Empirical Regularities in the Behavior of the Foreign Exchange Market. Carnegie-Rochester Conference Series on Public Policy, 11, 9-57, https://doi.org/10.1016/01 67-2231(79)90034-4

Nag, A. K., Mitra, A. (1998). Exchange Rate of the Rupee and Purchasing Power Parity. Economic and Political Weekly, 33(25), 1525-1532.

Olgu, Ö. (2011). European Banking: Enlargement, Structural Changes and Recent Developments. London: Palgrave Macmillan, p. 263.

Polak, J. J. (1957). Monetary Analysis of Income Formation and Payments Problems. Staff Papers (International Monetary Fund), 6, 1-50. https://doi.org/10.2307/3866128

Risk Management in Banking

Rogoff, K. (1996). The Purchasing Power Parity Puzzle. Journal of Economic Literature, 34(2), 647-668.

Saxa, B. (2014). Forecasting Mortgages: Internet Search Data as a Proxy for Mortgage Credit Demand. Czech National Bank. Working Paper No. 2014/14.

Schlueter, T., Sievers, S., Hartmann-Wendels, T. (2015). Bank Funding Stability, Pricing Strategies and the Guidance of Depositors. Journal of Banking \& Finance, 51, 43-61, https://doi. org/10.1016/j.jbankfin.2014.10.007

Sharma, S. C., Kandil, M., Chaisrisawatsuk, S. (2005). Currency Substitution in Asian Countries. Journal of Asian Economics, 16(3), 489-532, https://doi.org/10.1016/j.asieco.2005.04.013

Stanovich, K. E., West, R. F. (2000). Individual Differences in Reasoning: Implications for the Rationality Debate? Behavioral and Brain Sciences, 23(5), 645-665, https://doi. org/10.1017/s0140525x00003435

Tobin, J. (1969). A General Equilibrium Approach to Monetary Theory. Journal of Money, Credit and Banking, 1(1), 15-29, https://doi.org/10.2307/1991374

Tversky, A., Kahneman, D. (1981). The Framing of Decisions and the Psychology of Choice. Science, 211(4481), 453-458, https://doi.org/10.1126/science.7455683

Vlastakis, N., Markellos, R. N. (2012). Information Demand and Stock Market Volatility. Journal of Banking \& Finance, 36(6), 1808-1821, https://doi.org/10.1016/j.jbankfin.2012.02.007 
Yang, X., Pan, B., Evans, J. A., Lv, B. (2015). Forecasting Chinese Tourist Volume with Search Engine Data. Tourism Management, 46, 386-397, https://doi.org/10.1016/j. tourman.2014.07.019

Yuan, C. (2011). The Exchange Rate and Macroeconomic Determinants: Time-Varying Transitional Dynamics. North American Journal of Economics and Finance, 22(2), 197-220, https://doi.org/10.1016/j.najef.2011.01.005

Zellner, A. (1986). On Assessing Prior Distributions and Bayesian Regression Analysis with G-Prior Distributions. In Bayesian Inference and Decision Techniques: Essays in Honor of Bruno de Finetti, ed. Goel, P. K., Zellner, A., 233-243. Amsterdam: North-Holland. ISBN 978-0-444-87712-3. 